\title{
CONVERSACIONES SOBRE ADMINISTRACIÓN Y ORGANIZACIONES EN LATINOAMÉRICA. UN ÉNFASIS EN EL ESTADO ACTUAL DE LA INVESTIGACIÓN Y LA EDUCACIÓN"
}

\author{
Carmen Alejandra Ocampo-Salazar*** \\ Mariano Gentilin**** \\ Diego René Gonzales-Miranda*****
}

* doi: 10.11144/Javeriana.cao29-52.caol. Este artículo es producto de la investigación “Revisión de literatura sobre el estudio de las organizaciones a nivel nacional y latinoamericano entre el 2000 y el 2014", financiado por la Universidad EAFIT desde 15 de enero de 2014, hasta 30 de diciembre de 2014. El artículo se recibió 03/04/2016 y se aprobó el 30/05/2016. Sugerencia de citación: Ocampo-Salazar, C. A.; Gentilin, M. y Gonzales-Miranda, D. R. (2016). Conversaciones sobre administración y organizaciones en Latinoamérica. Un énfasis en el estado actual de la investigación y la educación. Cuadernos de Administración, 29 (52), 13-51. http://dx.doi.org/10.11144/Javeriana.cao29-52.caol.

** Ph.D. (C) en Administración de la Universidad EAFIT, Medellín, Colombia. Profesora de Organización y Gerencia, Escuela de Administración de la Universidad EAFIT, Medellín, Colombia.

Correo electrónico: cocampo2@eafit.edu.co

*** Doctor en Estudios Organizacionales (C), de la Universidad Autónoma Metropolitana, México DF, México. Ph.D. (C) en Administración, de la Universidad EAFIT, Medellín, Colombia. Profesor de Organización y Gerencia, Escuela de Administración de la Universidad EAFIT, Medellín, Colombia.

Correo electrónico: mgentilin@eafit.edu.co

**** Doctor en Estudios Organizacionales de la Universidad Autónoma Metropolitana, México D.F., México. Ph.D. en Administración, de la Universidad EAFIT, Medellín, Colombia. Profesor de Organización y Gerencia, Escuela de Administración de la Universidad EAFIT, Medellín, Colombia.

Correo electrónico:dgonzal8@eafit.edu.co 


\section{Conversaciones sobre administración y organizaciones en Latinoamérica. Un énfasis en el estado actual de la investigación y la educación}

Conversations on management and organizations in Latin America. An emphasis on the current status of research and education

\section{ResUMeN}

Este artículo presenta diferentes conversaciones sobre administración y organizaciones que se han publicado en diez de las principales revistas latinoamericanas en el periodo 2000-2014. La unidad de análisis fueron 3.210 artículos clasificados en cuatro ejes conceptuales: Teoría Administrativa, Teoría de la Organización, Estudios Organizacionales e Investigación y Educación. Para la categorización se utilizó el software Atlas. ti, versión 7.5, complementado con métodos de análisis de contenido y frecuencia. Se identificaron tendencias temáticas, los autores que más publican y las redes de cooperación, así como también líneas de investigación y educación en dicho campo. Los resultados de la investigación invitan a reflexionar acerca de los fundamentos de la administración y las implicaciones de sus intervenciones en las organizaciones.

Palabras clave: administración, organización, estudios organizacionales, investigación y educación.

Clasificación JEL: Moo, Mıo

\section{ABSTRACT}

This paper presents different conversations on management and organizations that have been published in ten of the main Latin American journals in the time period 2000-2014. The unit of analysis consisted of 3.210 papers classified in four conceptual axes: Management Theory, Organization Theory, Organizational Studies, and Research and Education in management and organizations. For the categorization, the Atlas.ti software, version 7.5, was used, complemented with content and frequency analysis methods. Thematic trends in management and organizations, the most prolific authors, the cooperation networks, as well as thoughts on research and education in this field were identified in this study. The results of the research invite to reflect on the basis of management and the implications of its interventions on organizations.

Keywords: Management, organization, organizational studies, research and education.

JEL Classification: Moo, M10

\author{
Conversas sobre \\ administração e \\ organizações na América \\ Latina. Uma ênfase no \\ estado atual da pesquisa e \\ da educação
}

\section{Resumo}

Este artigo apresenta diferentes conversas sobre administração e organizações que se publicaram em dez das principais revistas latino-americanas no período 2000-2014. A unidade de análise foram 3.210 artigos classificados em quatro eixos conceituais: Teoria Administrativa, Teoria da Organização, Estudos Organizacionais e Pesquisa e Educação. Para a categorização, utilizou-se o software Atlas.ti, versão 7.5, complementado com métodos de análise de conteúdo e frequência. Identificaram-se tendências temáticas, os autores que mais publicam e as redes de cooperação, bem como também linhas de pesquisa e educação nesse campo. Os resultados da pesquisa convidam a refletir acerca dos fundamentos da administração e as implicações de suas intervenções nas organizações.

Palavras-chave: administração, organização, estudos organizacionais, pesquisa e educação.

Classificação JEL: Moo, M10 


\section{Introducción}

En general, una conversación se entiende como un diálogo en el que se intercambian ideas sobre un tema con el fin de reflejar, reproducir y refutar otras conversaciones. Toda conversación está condicionada por el contexto de los interlocutores al momento de construir su discurso. En el estudio de las organizaciones, el concepto de conversaciones se ha utilizado en un sentido de metáfora, para referenciar la manera en que se construye el campo de estudio, a partir de conversaciones entre investigadores sujetos a paradigmas, métodos y supuestos que se derivan de otras conversaciones (Clegg y Hardy, 1996).

Se reconocen diversas transformaciones empresariales y organizacionales en el ámbito latinoamericano desde la década de 1990, dado el incremento de diálogos sobre los retos en las escuelas de administración (Malaver, 1999), abocadas a enfrentar un entorno global y digital que exigió cambios para la formación académica y gerencial. Nuevas corrientes de estudio como los Critical Management Studies (CMS) y los Estudios Organizacionales (EO), propiciaron un aumento de críticas y desafíos frente a una educación mercantilizada, métodos de enseñanza obsoletos, visiones reducidas de la gestión, instrumentalización del conocimiento y ausencia de una pedagogía de pensamiento crítico y analítico (Paes y Rodrigues, 2006).

Se reconocieron otras voces en el ámbito de la administración y las organizaciones en la década del 2000, caracterizadas por una profunda reflexión dado los fuertes escándalos que enfrentó el mundo de los negocios, entre ellos: Enron, Parmalat y WorldCom. Los diversos debates en la academia estuvieron enmarcados en una dimensión ética del discurso (Wood y Paes, 2004), que cuestionó las exigencias en el entorno gerencial del más alto nivel y la falta de integración del enfoque moral y ambiental en su formación.

Las críticas se han extendido también a la investigación en administración por la falta de rigor para generar conocimiento relevante y original. En particular en Latinoamérica se identifican desafíos para la investigación en administración, debido a la orientación práctica del campo (Calderón y Castaño, 2005). Por ello, se revisó la producción científica en el ámbito latinoamericano en diez de las principales revistas indexadas en las base de datos Scopus, con el objetivo de analizar las conversaciones publicadas e identificar tendencias temáticas, retos y perspectivas de investigación y educación en el campo de la administración y la organización, en un periodo de estudio de quince años (2000-2014). En total se consideraron 3.210 artículos, escritos por 5.336 autores, pertenecientes a 1.096 instituciones de 78 países. 
En consecuencia, se identificó que la literatura relacionada con el estudio de la administración y las organizaciones en Latinoamérica evidencia dos tendencias teóricas: una de carácter más funcional y prescriptiva y otra con una mirada comprensiva y crítica. Lo funcional se entiende como aquella aproximación que busca el desarrollo eficiente de los procesos administrativos para cumplir con los objetivos planteados. Es un enfoque que está mediado por una epistemología positivista y pragmática, que ve a las organizaciones como estructuras de funciones efectivas que propician el funcionamiento eficaz de la organización (Donaldson, 2003). Lo crítico y comprensivo, en cambio, propicia una autorreflexión sobre el actuar y sus consecuencias, lo cual articula, generalmente, un trabajo interdisciplinario con las ciencias sociales. Esta perspectiva se opone y denuncia a aquellas acciones que imperan injustamente en la sociedad, al reducir el pensamiento al positivismo, y la razón a la razón subjetiva (Horkheimer, 2002), mediante una postura que busca ante todo, comprender la realidad -y con ello las organizaciones-, antes que intentar resolver los problemas que la aquejan.

El ejercicio que aquí se presenta se realizó a partir de los marcos teóricos definidos para la investigación, y fueron a su vez, sustentados por los resultados del análisis de los artículos. Así, se pudieron clasificar los documentos en los cuatro ejes conceptuales que concentran el campo de estudio. A partir de ello, se pudo reconocer que en la Teoría Administrativa (TA) y la Teoría Organizacional (T0) se concentraron aquellos trabajos que tienen relación con una perspectiva funcional, mientras que otros artículos coincidían con una postura crítica relacionados con los Estudios Organizacionales (E0) e Investigación y Educación en administración y organizaciones (I\&E). Como parte de la contribución de este artículo, se hace mayor énfasis en el análisis de este último eje conceptual, con el fin de exponer el estado actual de las conversaciones en materia de investigación y educación, y de plantear reflexiones y desafíos para los investigadores y la academia en general.

El desarrollo del trabajo buscó responder las siguientes preguntas: ¿cuál es el eje conceptual predominante en las conversaciones objeto de análisis?, ¿qué temáticas están estudiando los investigadores en administración y organizaciones por eje conceptual?, ¿cuáles son los autores que más publican y qué redes de estudio se identifican en los textos analizados?, ¿cuáles reflexiones se han generado en la educación y la investigación de la administración y las organizaciones? En las respuestas se reconoce la preeminencia que tiene la mirada funcional y pragmática en el estudio administrativo y organizacional en Latinoamérica, la importancia de publicar en coautoría y las redes de colaboración que se han construido entre autores e instituciones; asimismo, se identifican las principales temáticas en cada eje conceptual y las reflexiones investigativas y educativas. 
El documento está dividido en cinco secciones: en la primera se explican los cuatro ejes conceptuales, a partir de los cuales se clasificaron los artículos de las revistas seleccionadas. En la segunda se exponen los aspectos metodológicos relacionados con la recolección de los datos y su análisis. En la tercera se analizan los resultados de la revisión. En la cuarta se discuten los resultados a partir del énfasis en la realidad de la investigación y la educación en el contexto latinoamericano. Por último, en la quinta sección se presentan las limitaciones del estudio y las futuras líneas de investigación.

\section{Marco conceptual}

Los ejes conceptuales utilizados para la clasificación de los artículos objeto de análisis surgieron a partir de una revisión teórica de los marcos existentes para el estudio de las organizaciones: TA, T0, E0 e I\&E. Este último congrega aquellos trabajos que proponen reflexiones alrededor de la investigación y la educación, así como los modelos y herramientas pedagógicas.

En América Latina el estudio de las organizaciones se ha caracterizado por una orientación técnico-instrumental y prescriptiva, relegando los problemas organizacionales a factores que se deben resolver según los criterios de eficiencia y productividad. A raíz de ello se ha configurado históricamente un isomorfismo organizacional en torno a un solo eje: la eficiencia de la empresa: “La empresa se convierte en el punto de referencia de la organización, y su lenguaje, métodos, herramientas y técnicas contaminan cada vez más el mundo organizacional" (Vieira y da Silva, 2011, p. 457).

Como discrepancia de lo anterior, Ailon (2006) postula que en las últimas dos décadas han surgido voces críticas que objetan las bases de la corriente principal del pensamiento organizativo y de gestión, a partir del cuestionamiento de textos canónicos. Esto se debe a la complejidad organizacional, y a que el estudio de las organizaciones está pasando por un periodo que favorece el análisis de distintos fenómenos desde variadas perspectivas (Martins y Barrera, 2012).

Esta diversidad de aproximaciones al estudio de las organizaciones se refleja en los cuatro ejes propuestos, que están sujetos a debates y controversias. Algunos autores, por ejemplo, establecen diferencias entre los tres primeros (Barba, 2013; Gonzales-Miranda, 2014; Gonzales-Miranda y Gentilin, 2013; Montaño, 2004; Ramírez, Vargas y De la Rosa, 2011); otros, en cambio, integran la TA, la TO y los EO en el concepto "paraguas" de Estudios Organizacionales (Sanabria, Saavedra y Smida, 2013), consideran de manera similar la 
TA y la T0 (Vieira y da Silva, 2011), la T0 y los E0 (Hatch, 1997; Tsoukas y Knudsen, 2005), o establecen una diferenciación radical entre la TA y la T0 (Scott, 2003).

La clasificación conceptual utilizada para este trabajo no pretende profundizar en la discusión-aún inacabada- sino proponer fundamentos teóricos que permitan distinguir un eje respecto del otro, en aras de clasificar los artículos y con ello identificar los retos en investigación y educación sobre la administración y las organizaciones. En ese orden de ideas no es menester exponer las diversas corrientes actuales que se desprenden de cada uno de estos marcos, ni mencionar a los autores seminales que las representan, tan sólo, exponer sus principales características con miras a contextualizar la tipología adoptada para el análisis de los datos.

\subsection{Teoría Administrativa}

La administración surgió primero, a partir de una serie de situaciones empíricas, según Jurado (2015), sin discurso propio. Por ello, dada esta naturaleza, la administración significa un saber administrativo, no una práctica en sí, pero es un saber administrativo en la práctica organizacional (De Mattos, 2009). Con el tiempo y la sistematización de los trabajos de algunos autores clásicos, como Taylor y Fayol, se originó una práctica discursiva, lo que se puede entender como un saber o teoría administrativa.

La administración ha sido objeto de diversas controversias en cuanto a su estatus científico (Podestá y Jurado, 2003; Bédard, 2003, 2004; Muñoz, 2011; Marín-Idárraga, 2012), su definición y delimitación (Aktouf, 1998; Dávila, 2001) y su objeto de estudio (Hernández, Saavedra y Sanabria, 2007; López, 1999). Sin entrar a ahondar en tales debates, la crítica se fundamenta sobre todo en su pragmatismo, lo cual reafirma su propósito funcional en la aplicación de modelos desarrollados en contextos muy diferentes a los de América Latina (Vieira y da Silva, 2011), que han sido acogidos como únicos, verdaderos y universales (Bédard, 2003), al punto de ser replicados sin someterlos a reflexión o cuestionamiento (Bédard, 2004).

Las prácticas administrativas, en su estatus ambivalente y aún controversial en el ámbito académico, tienen su fundamento en la razón, en tanto categoría filosófica que ha oscilado entre dos lógicas: una de tipo objetivo, en la que el observador es un ente externo, y otra, de tipo subjetivo, que se sirve del interés particular de la técnica con un propósito instrumental-utilitario (Marín, 2007). En la última se entiende a la administración como un saber orientado a la práctica. 
Dicha racionalidad ha acompañado las propuestas de los pensadores y promotores más representativos de la TA, como lo fueron Taylor (1961) y Fayol (2003), quienes reconocieron en el ser humano su deseo de maximizar las ganancias, y su capacidad administrativa y profesional. La TA se inscribe así en una orientación de tipo prescriptivo, porque busca la aplicación de los conceptos y métodos para alcanzar productividad y eficacia, con una clara tendencia a universalizar sus resultados. Dicha aproximación está relacionada con las investigaciones en las organizaciones, al centrar su atención en las actividades administrativas y en las funciones, a la vez que estructura la base material para su estudio, al considerarlas un objeto productivo moderno centrado en la rentabilidad y en la producción.

En la TA la conceptualización y la teoría parecerían ir unos pasos atrás de la práctica y la acción, lo que fomenta excesivo pragmatismo, y consecuente hostilidad a la actividad intelectual (Dávila, 2005). No obstante, se reconoce un avance en la conformación de un corpus de conocimiento que ha dado lugar al surgimiento de diversos temas como estrategia, liderazgo, mercadeo, finanzas, gestión humana, gestión de proyectos, entre otros.

\subsection{Teoría de la Organización}

En la década de 1930 los trabajos de Mayo (1880-1949) darían inicio a una nueva corriente de estudio de la administración y las organizaciones denominada "ciencias del comportamiento organizacional", donde se buscó dar herramientas instrumentales al administrador con el objetivo de crear y mantener un clima laboral propicio para poder influenciar a las personas, y generar mayor productividad y eficiencia (Aktouf, 1998). Esta aparente preocupación por lo humano marcó el inicio -entre 1925 y 1945- de la escuela de las relaciones humanas, a partir de la cual surgió la T0 (Ibarra y Montaño, 1990).

El desarrollo de la T0 propició la inclusión de diversas áreas del saber, como la filosofía, la sociología, la antropología y la psicología, al estudio de las organizaciones (Ibarra, 2006). La TO se concibe así como la consciencia teórica de la TA, debido a su continua búsqueda de sentido para enfrentar problemas de las organizaciones modernas, siempre ligadas a los mismos fines: productividad, eficiencia, eficacia y control (De la Rosa, 2012).

La TA se gestó en un contexto apremiado por las inventivas y la tecnología, regido por una visión mecanicista que impedía ver el carácter humano de los trabajadores (Bendix, en Jurado, 2015). La T0, en cambio, propició la inclusión del obrero, la interacción humana y la informalidad como unidad de análisis (Mayo, 1972), al reconocer la organización 
industrial como un sistema sociotécnico. Sin embargo, el tema de la racionalización subjetiva vigente en la TA, hizo presencia en la T0. Esta racionalización tuvo una fuerte influencia en el desarrollo conceptual del análisis organizacional. Así, “la organización se convierte en una herramienta o instrumento para la autorización y realización de metas colectivas a través del diseño y la gestión de estructuras dirigidas por la administración y la manipulación del comportamiento organizacional" (Reed, 1996, p. 36).

La T0 se consolidó, a la vez que marcó una diferencia con la TA, a partir de los estudios de Woodward (1975), Pugh (1997), Burns y Stalker (1961) y Lawrence y Lorsch (1973), quienes dieron lugar en la década de 1960 al movimiento de la contingencia. Al estudiar el entorno la organización quedó como objeto de estudio en sí mismo y por primera vez se evidenciaron aspectos de singularidad espacial y temporal que contradicen la pretensión de un discurso único y general. Esto significó un quiebre respecto de la TA, al contraponer la expresión "all depends", que cuestiona la máxima taylorista del "one best way" (Clegg, 1990), debido a que no hay una mejor forma de hacer las cosas sino tantas formas como organizaciones existan (Montaño, 1994).

En dicho eje conceptual se relacionan temas como la escuela de las relaciones humanas, la burocracia, la escuela de comportamiento, el movimiento de la contingencia, escuela de toma de decisiones, nuevas relaciones humanas y desarrollo organizacional, entre otros.

\subsection{Estudios Organizacionales}

El campo de los EO se puede considerar como un eje conceptual relativamente nuevo en América Latina. Algunos autores indican que su inicio se remonta a la década de 1970 con la constitución de EGOS (European Group for Organizational Studies) y de la revista Organización Studies (Clegg y Bailey, 2008). Otros ubican su origen en el periodo posterior a la Segunda Guerra Mundial (Augier, March y Ni Sullivan, 2005; Scott, 2004) o a partir del movimiento de la contingencia (Gonzales-Miranda, 2014).

La transición de la T0 a los E0 estuvo mediada por un factor crítico-reflexivo que permitió proponer nuevos enfoques para la comprensión del campo organizacional, pero sin excluir a la T0, sino que, por el contrario, busca recuperar sus propuestas. Este fue un proyecto de origen europeo que fomenta el pensamiento crítico (Montaño, 2004) y surgió como una respuesta alternativa a la T0 norteamericana (Shenhav, 2003), que consideró como objeto de estudio una organización lineal, estática y causal (Ibarra, 2006). 
En los E0 se busca generar conocimiento propio y específico del contexto en el que se encuentran insertas nuestras organizaciones. "Es indispensable interpretar los problemas de organización de nuestras realidades locales" (Ibarra, 2006, p. 145) o recuperar de forma crítica la T0 al pensar la realidad de Latinoamérica (Ibarra y Montaño, 1992). Por ello, el gran rompimiento paradigmático consistió en intentar entender las prácticas y productos teóricos en función de los contextos sociales y culturales de los actores involucrados (De Mattos, 2009), para buscar comprender -más que solucionar- los fenómenos sociales al interior de las organizaciones (Clegg y Dunkerley, 1977).

En este eje conceptual se vinculan temas como poder, teoría crítica y posmodernismo, cultura en las organizaciones, nuevo institucionalismo, antropología de las organizaciones y aprendizaje, psicoanálisis organizacional, Critical Management Studies (CME), ecología de las organizaciones, teoría de la ambigüedad organizativa y conocimiento organizacional, entre otros.

\subsection{Investigación y Educación en administración y organizaciones}

La investigación y la educación, como eje histórico y articulador en el campo administrativo y organizacional, han estado asociadas con la producción y la generación de conocimiento, así como con la reflexión y las metodologías con las que se construye el saber. En este sentido, la investigación se puede entender como una actividad social que busca contribuir a un campo de conocimiento, aunque, en esencia, ayuda a resolver problemas sociales a partir de la comprensión de la realidad (Calderón y Castaño, 2005). La educación, entendida en un nivel superior, hace referencia a la reflexión, la problematización, la discusión, la argumentación, y la construcción del conocimiento (Marín, 2005).

Las dos actividades están íntimamente relacionadas: la investigación ha constituido un aspecto clave en la educación y en la universidad como punto de encuentro; en este sentido, una educación de calidad debería soportarse en la investigación (Malaver, 1999). Por su parte, la enseñanza “debe motivar la participación, buscando que la discusión y el debate contribuyan a la incorporación del conocimiento" (Marín, 2005, p. 53), así como a la generación de ideas para nuevos proyectos de investigación por parte de los docentes implicados (Duque y Royuela, 2005). “Sin la renovación del conocimiento, los programas académicos no podrían avanzar; la enseñanza ayuda a identificar temas para la investigación" (Daniels, 1999). 
En administración, la investigación no ha sido tradicionalmente una fortaleza, debido a la gran orientación práctica y al consecuente descuido por lo teórico (Calderón y Castaño, 2005). “La tentación de pretender cambiar el mundo, subyuga mucho más que la ardua tarea de intentar comprenderlo" (Dávila, 2005, p. 40). En cuanto a la educación, los procesos de enseñanza-aprendizaje deben orientarse a la socialización del conocimiento, con énfasis en los aspecto pedagógicos, de tal manera que se posibilite hacer frente a las necesidades de la sociedad actual (Marín, 2005). La pedagogía, que en términos generales tiene por objeto analizar y comprender el fenómeno de la educación, ha intervenido en la discusión de la relación entre teoría y proceso de transmisión del conocimiento, con énfasis en las técnicas que se utilizan en el aula (Wren, Buckley y Michaelsen, 1994), así como en la excesiva instrumentalización de las mismas (0ttewill, 2003).

La investigación y la educación, en tantos ejes articuladores, deberían privilegiar la reflexión sobre sí mismas. Es decir, incentivar los estudios que posibiliten miradas críticas en torno a aspectos relacionados con qué y cómo se investiga, acerca de qué y cómo se enseña, y qué y cómo se emplean los métodos de enseñanza en administración y organizaciones, lo que implica pensar las posibilidades acerca de cómo se crea y reproduce el conocimiento en dichos procesos (Chia y Holt, 2008). Por ello, este eje busca identificar las contribuciones científicas que, insertas en el ámbito administrativo y organizacional, tienen como temáticas principales la investigación, la educación y la pedagogía.

\section{Aspectos metodológicos}

El objetivo general de la investigación fue analizar las conversaciones publicadas sobre administración y organizaciones en las principales revistas latinoamericanas con el fin de mostrar tendencias temáticas, retos y perspectivas de investigación y educación en el campo de la administración y los estudios de la organización entre 2000 y 2014 . Para el cumplimiento de este objetivo se trazó un proceso metodológico que incluyó recolección de los datos, análisis de la información y categorización.

\subsection{Recolección de los datos}

Se predefinieron las revistas para ser analizadas con el fin de identificar artículos académicos de impacto internacional que proveen indicadores basados en metodologías de citación con temáticas que son útiles y razonablemente válidas para el análisis en términos de insumos para la investigación (Garfield, 1972). Además, se tuvo presente que dichos artículos hubiesen sido sometidos a un proceso de revisión antes de su publicación, 
más que los libros o capítulos de libros (Ullah, Butt y Haroon, 2008) -sin decir con ello que los libros sean menos importantes-, lo cual garantiza su relevancia para el análisis.

Para la selección de las revistas se utilizó la base de datos Scopus, que evalúa el impacto que tienen los artículos por la cantidad de referencias citadas en un campo temático específico. Tres criterios fueron relevantes en este proceso: (1) categorías temáticas relacionadas con la investigación (organization, business, management y sociology, entre otras); (2) el indicador SJR (Scimago Journal \& Country Rank) y (3) los rangos intercuartiles de la distribución de frecuencia de los respectivos indicadores. Las diez revistas seleccionadas se encontraban entre el tercer (03) y el valor final de la distribución (04) para el año 2014 en Scopus.

\section{Tabla 1}

Revistas seleccionadas para la investigación

\begin{tabular}{|c|c|c|c|c|}
\hline Revista & País & Número de artículos & Posición & SJR \\
\hline Journal of Technology Management \& Innovation & Chile & 451 & Q3 & 0,25 \\
\hline Revista Venezolana de Gerencia & Venezuela & 419 & Q3 & 0,20 \\
\hline $\begin{array}{l}\text { Journal of Theoretical \& Applied Electronic Commerce } \\
\text { Research }\end{array}$ & Chile & 197 & Q3 & 0,21 \\
\hline RAE. Revista de Administração de Empresas & Brasil & 386 & Q3 & 0,21 \\
\hline Innovar. Revista de Ciencias Administrativas y Sociales & Colombia & 473 & Q3-04 & 0,19 \\
\hline BAR. Brazilian Administration Review & Brasil & 207 & Q3 & 0,17 \\
\hline RBGN. Revista Brasileira de Gestão de Negócios & Brasil & 337 & Q3-Q4 & 0.13 \\
\hline Reforma y Democracia. Revista del CLAD & Venezuela & 330 & Q4 & 0,12 \\
\hline Academia. Revista Latinoamericana de Administración & Colombia & 140 & Q4 & 0,12 \\
\hline Cuadernos de Administración & Colombia & 270 & Q4 & 0,11 \\
\hline Total de artículos & & 3.210 & & \\
\hline
\end{tabular}

Fuente: elaboración propia con base en los datos consultados en el indicador SJR.

En la revisión de los textos sólo se relevaron publicaciones que tienen carácter de artículo, sin considerar comentarios del editor, foros, reseñas de libros, estudios de caso y otros. En total se analizaron 3.210 artículos.

\subsection{Análisis de la información}

En el análisis de los artículos se tuvieron presente datos como título, nombre(s) del(os) autor(es), institución en la que trabaja(n), resumen y palabras clave, con el fin de clasificar 
las temáticas centrales en cada uno de los ejes conceptuales: TA, T0, E0 e I\&E. Después se analizaron en profundidad los textos relacionados en el último eje y se identificaron retos y desafíos para el campo de la administración y las organizaciones.

Siguiendo a Tranfield, Denyer y Smart (2003) se buscó una consolidación conceptual de las diversas temáticas estudiadas en y alrededor de las organizaciones, en un campo fragmentado que no está exento de debates y controversias que sirven en la construcción de conocimiento.

\subsection{Categorización}

La categorización y la identificación de las principales temáticas se fundamentaron en el marco conceptual y en la interpretación y conceptualización de los investigadores para clasificar un determinado texto en la categoría correspondiente gracias al uso de herramientas de análisis de contenido. En ese sentido, el estudio realizado se inscribe en una metodología cualitativa, caracterizada por un procedimiento más inductivo que deductivo y con una mirada panorámica y de concreción (Hancké, 2009; Shankar, 2009).

Como herramienta metodológica para la categorización se utilizó el software Atlas.ti, versión 7.5. Este programa, a diferencia de otros como Ethnograph, HyperResearch y Nudist, permite el análisis de información con alto manejo de texto, notas y conceptos teóricos. En el programa las categorías principales que se relacionan se determinaron a partir de los ejes conceptuales: TA, TO, EO e I\&E. Ver figura 1.

Una vez clasificados los artículos se procedió con el análisis del eje I\&E al relacionar cada una de las subcategorías, como se muestra en la figura 2.

La interpretación de los datos exige precisar que el software empleado relaciona unos números $\left\{\mathrm{N}^{\circ} 1-\mathrm{N}^{\circ} 2\right\}$. El $\mathrm{N}^{\circ} 1$ indica la cantidad de artículos en cada categoría y representa la relevancia, y, por lo tanto, su validez. El $\mathrm{N}^{\circ} 2$ indica las subcategorías evidenciadas a partir de la estructura que se va creando y expresa el grado de densidad teorética de la misma. Por ejemplo, en el eje conceptual IE se relacionaron 160 artículos con tres subcategorías: educación, investigación y pedagogía; en cada una de ellas se indica el número de artículos en los que se identificó, para el caso 80,55 y 25, en su orden. 


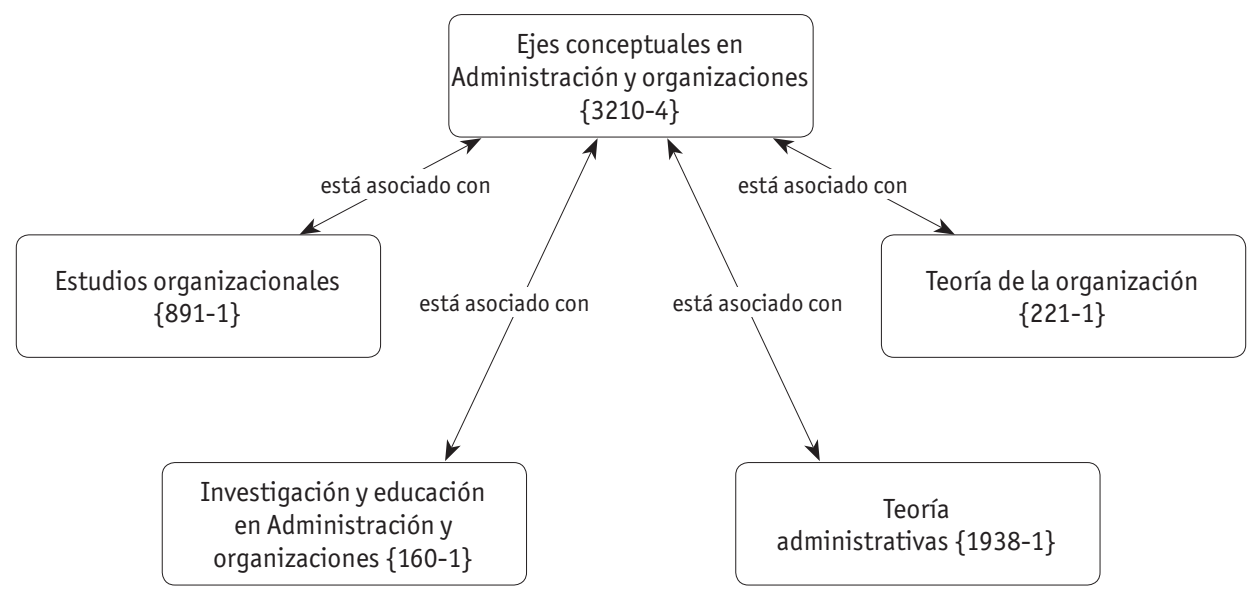

Figura 1. Número de artículo por ejes conceptuales

Fuente: elaboración propia a partir de los datos procesado en el software Atlas.ti

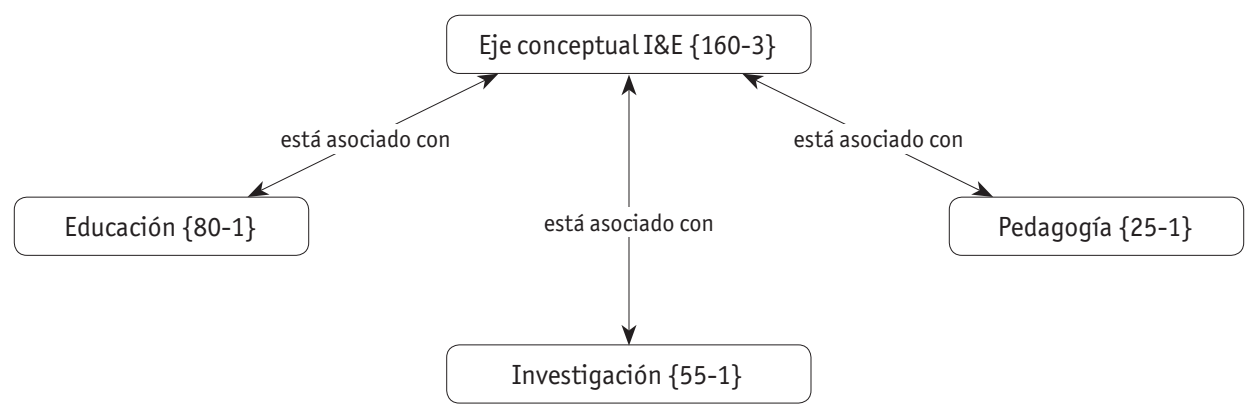

Figura 2. Temáticas en el eje conceptual I\&E

Fuente: elaboración propia a partir de los datos procesado en el software Atlas.ti

\section{Resultados}

El análisis de la información se presenta a partir de las siguientes subsecciones: 1. Administración y organizaciones: panorama general de los últimos 15 años, que relaciona las temáticas, los autores que más publican y las redes de cooperación; 2. Principales temáticas por ejes conceptuales, en las que se destacan aspectos relevantes de las principales temáticas que fueron identificadas; 3 . Administración y organizaciones: conversaciones sobre investigación y educación, que enfatiza en las problemáticas y desafíos que se evidencian en Latinoamérica respecto al eje I\&E. 


\subsection{Administración y organizaciones: panorama general de los últimos 15 años}

Este apartado relaciona las temáticas por eje conceptual (TA, TO, E0 e I\&E), los autores con más publicaciones y las redes de cooperación.

3.1.1. EN RELACIÓn CON LAS TEMÁTICAS POR EJE CONCEPTUAL. En los artículos objeto de estudio se observó el abordaje de diversas temáticas relacionadas con la administración y las organizaciones. En este sentido, se identificaron dos enfoques predominantes en la literatura revisada: uno funcional, en el cual se incluyen los ejes TA y T0, y otro comprensivo, que contiene los ejes EO e I\&E.

La siguiente tabla expone el total de artículos que fueron clasificados en cada uno de los ejes conceptuales de la investigación:

\section{Tabla 2}

Cantidad de artículos por eje conceptual

\begin{tabular}{lcc}
\hline \multicolumn{1}{c}{ Eje conceptual } & Número de artículos & Porcentaje \\
\hline Teoría Administrativa (TA) & 1.938 & 60 \\
Estudios Organizacionales (EO) & 891 & 28 \\
Teoría de la Organización (T0) & 221 & 7 \\
Investigación y Educación en administración y organizaciones (I\&E) & 160 & 5 \\
\hline Total de artículos & 3.210 & 100 \\
\hline
\end{tabular}

Fuente: elaboración propia

A partir de la clasificación de los artículos se evidencia que la TA prima como eje conceptual, con $60 \%$, seguida de los E0, con $28 \%$, así como de la T0 y la I\&E, con $7 \%$ y $5 \%$, en su orden. Se observa que la tendencia en las conversaciones sobre administración y organizaciones en América Latina ha sido más funcional que comprensiva. En este sentido, los resultados del estudio permiten interrogar el posicionamiento de los académicos en relación con los ejes críticos del análisis (E0 e I\&E), así como el anclaje de la academia en la TA y la TO con enfoque funcionalista.

Los hallazgos muestran la fuerte influencia que tiene la escuela anglosajona y su orientación pragmática en el campo de la administración y la organización. El profesor Diego Marín (2005) cita a Mardones para señalar que esta problemática es, en general, la misma que enfrentan las ciencias sociales en relación con las posturas y explicaciones que surgen 
según el canon de la ciencia (positivismo) para legitimarse científicamente, respecto a otras posturas de corte más comprensivo y crítico.

A partir de lo anterior, es posible señalar la necesidad de estudiar situaciones desde la perspectiva de los E0 para generar otras comprensiones de la realidad en el contexto latinoamericano, con el uso de temáticas como la teoría crítica y posmoderna, el aprendizaje y el conocimiento organizacional, la cultura, el neoinstitucionalismo y la sociología, entre otras, sin desacreditar las investigaciones funcionales que buscan la practicidad de los elementos administrativos en las organizaciones, necesarios, pero no suficientes, para la comprensión de las organizaciones, ni para fomentar la discusión en la academia. Algunos textos muestran dicho desafío al presentar las controversias y diferencias en el mencionado campo de conocimiento, que permite "una nueva perspectiva para la comprensión de los fenómenos organizacionales, cuya riqueza se encuentra en esa pluridisciplinariedad y en su perspectiva crítica, aspectos relevantes tanto para la investigación como para la intervención organizacional" (Gonzales-Miranda, 2014, p. 55).

El profesor Vizeu (2010) indica que la tendencia positivista en la investigación obstaculiza el análisis y la comprensión de la realidad administrativa y organizacional en el entorno local, y a partir de allí propone fortalecer la literatura según los E0 con trabajos que retomen áreas de las ciencias sociales, como la historia, por ejemplo. También Peci (2003) establece que se deben utilizar perspectivas sociológicas en la comprensión de las organizaciones, y desde la óptica de dos autores clásicos (Giddens y Bourdieu) muestra una síntesis del debate objetividad-subjetividad y la aplicabilidad de dichas perspectivas en el campo de los E0. Otras críticas similares se observan en Betancourt (2003), Contreras y Crespo (2005), Batista-dos-Santos, Mata de Lima y Holanda (2010), Vieira y da Silva (2011) y Siegler, Biazzin y Rodrigues (2014), quienes opinan que las publicaciones son insuficientes para proyectar marcos teóricos y conceptuales que posibiliten avances significativos para la academia en administración.

3.1.2. EN RELACIÓN CON LA AUTORÍA. En el panorama de los datos analizados se identificaron 5.336 autores adscritos a diferentes instituciones latinoamericanas e internacionales. En la tabla 3 se muestran los diez autores que más han publicado en las revistas objeto de análisis y la institución de adscripción.

De los 108 artículos que fueron escritos por quienes más han publicado en las revistas seleccionadas, se resalta que el $76 \%$ fueron en coautoría, lo que permite evidenciar una tendencia al trabajo grupal e interdisciplinario que se refleja en la correlación positiva 
entre la cantidad de textos publicados y la coautoría. También, se resalta que los datos generales de la investigación (figura 3) muestran la misma dinámica con la coautoría.

\section{Tabla 3}

\section{Autores e instituciones de origen}

\begin{tabular}{lc}
\hline \multicolumn{1}{c}{ Autor (institución) } & Número de artículos \\
\hline Gregorio Calderón Hernández (Universidad Nacional de Colombia / Universidad de Manizales) & 18 \\
Yuri Gorbaneff (Pontificia Universidad Javeriana / Institutos y Centros de Investigación) & 12 \\
Bernardo Kliksberg (Sector bancario / Sector Público / Consultor) & 11 \\
Flávio Carvalho de Vasconcelos (Fundação Getulio Vargas - FGV) & 11 \\
Eduardo Torres-Moraga (Universidad de Chile) & 10 \\
Edison Jair Duque-Oliva (Universidad Nacional de Colombia) & 10 \\
Florentino Malaver Rodríguez (Pontificia Universidad Javeriana / Universidad Nacional de & 9 \\
Colombia) & 9 \\
Roberto Sbragia (Universidade de São Paulo - USP / Universidade Paulista) & 9 \\
Juan David Velásquez Henao (Universidad Nacional de Colombia) & 9 \\
Luiz Artur Ledur Brito (Fundação Getulio Vargas - FGV) & 108 \\
\hline Total & 9 \\
\hline
\end{tabular}

Fuente: elaboración propia.

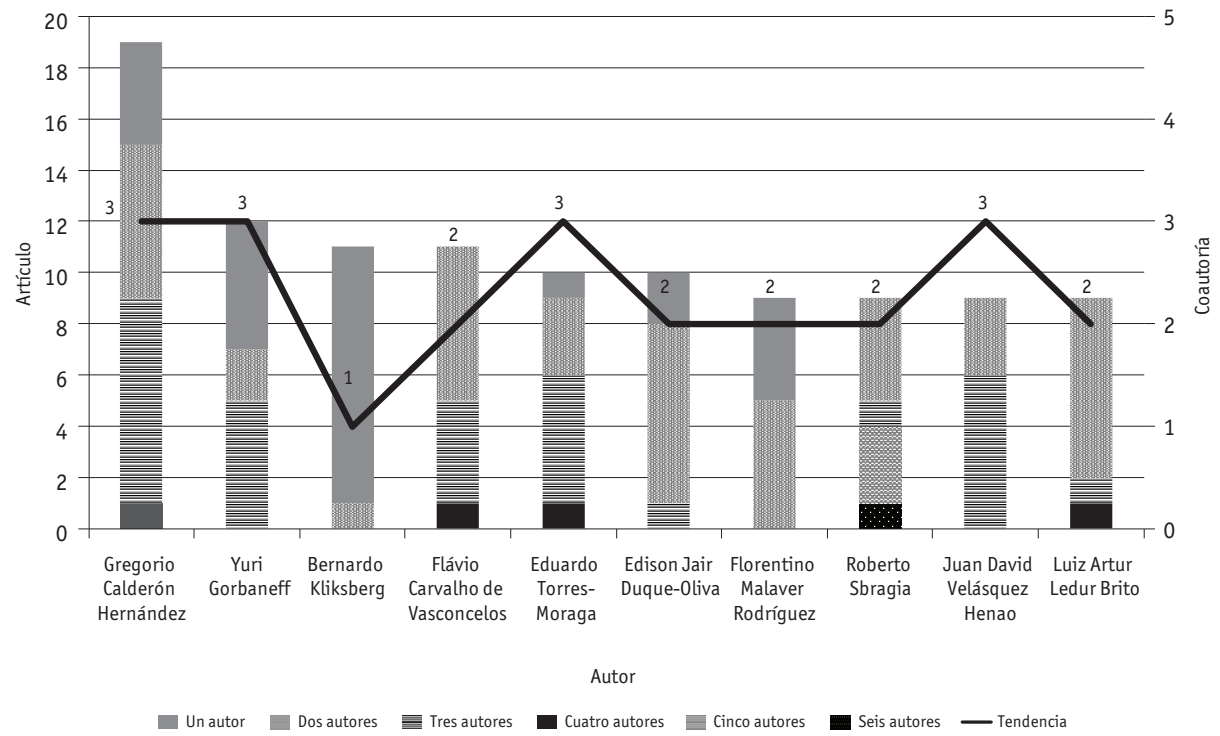

\section{Figura 3. Comparación de autoría por año}

Fuente: elaboración propia. 
La figura 4 también muestra un análisis lineal que permite determinar que los datos de la autoría única decrecen, en tanto que los de la compartida aumentan, con un punto de inflexión en el año 2007.

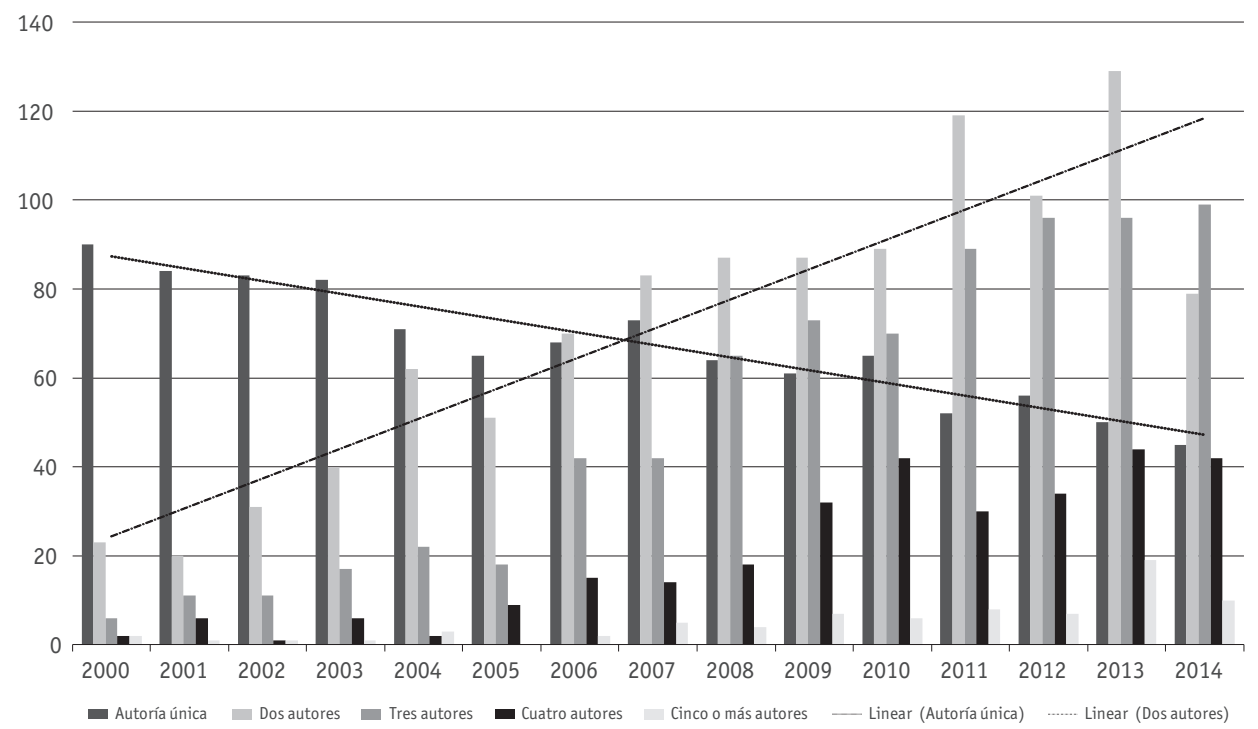

Figura 4. Comparación de autoría por año

Fuente: elaboración propia.

3.1.3 EN RELACIÓN CON LAS REDES DE COOPERACIÓN. No se puede comprender el análisis anterior sin considerar la generación de redes en los ámbitos latinoamericano e internacional porque las redes son una de las formas mediante las cuales se fomenta el trabajo interdisciplinario, el apoyo a la investigación desde diferentes instituciones y la cooperación para la publicación. Se reconoce, entonces, que el conocimiento generado por los diversos autores constituye un avance para el desarrollo de las comunidades científicas y del esfuerzo colectivo, en el que bien valdría identificar la interacción y la participación que se da entre los países de acuerdo con la adscripción de la institución de los autores. En total se identificaron 78 países relacionados con las publicaciones analizadas; los cinco primeros fueron: Brasil, con 36\%, seguido de España, con 14\%, Colombia, con 12\%, Venezuela, con $8 \%$, y Chile, con $5 \%$. 


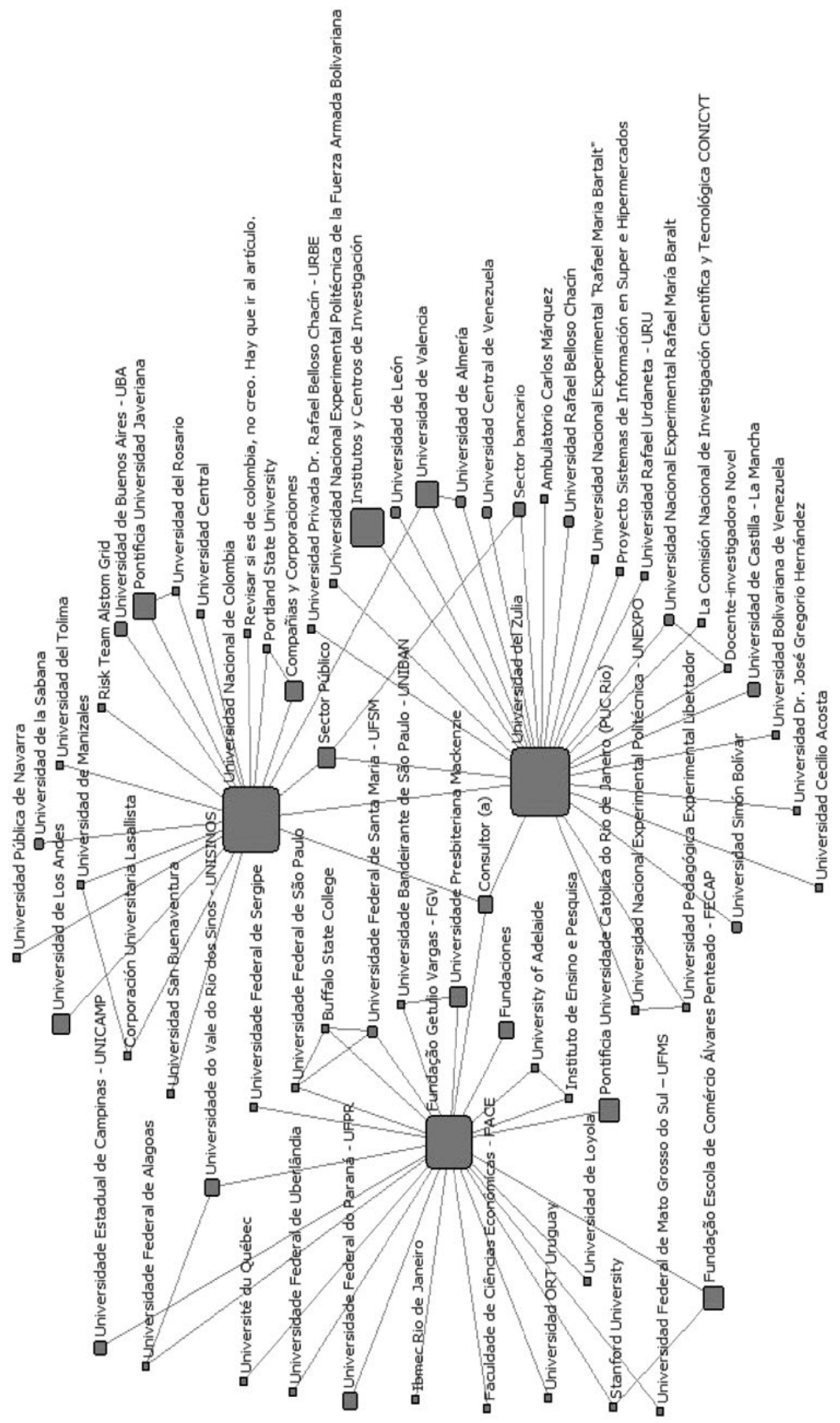

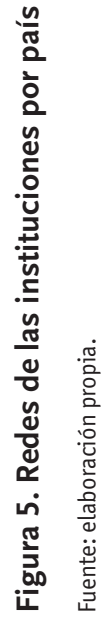


Respecto a las instituciones, se identificaron 1.096 que fueron informadas por los autores en las revistas analizadas. La cifra se obtiene de la cantidad de veces que una institución aparece mencionada como lugar de adscripción de los artículos. En ese sentido, un escrito puede relacionarse con varias instituciones. Si se tiene en cuenta lo anterior, las instituciones educativas que más artículos publicaron fueron la Universidad Nacional de Colombia (4,8\%), la Fundación Getulio Vargas de Brasil (3,9\%) y la Universidad de Zulia de Venezuela $(3,7 \%)$. En el análisis de redes de la figura 5 se destacan dichas universidades, ya que el tamaño de los nodos representa la cantidad de participación de las instituciones en artículos por medio de autores adscritos a las mismas. Así, y a manera de ejemplo, el 87\% -en promedio- de los autores que publican en las revistas brasileras (BAR, RAE, RBGN) son de Brasil. Esto da cuenta de cierto regionalismo académico en las publicaciones y de la poca participación de investigadores por fuera de la región que participan en estas redes.

\subsection{Principales temáticas por ejes conceptuales}

El análisis de las perspectivas de estudio de la administración y las organizaciones en Latinoamérica permitió realizar una clasificación, no agotada, de las temáticas que corresponden a cada uno de los ejes (TA, TO, EO e I\&E). A continuación, en la tabla $\mathrm{N}^{\circ} 4 \mathrm{se}$ presenta el panorama evidenciado, y posteriormente se discuten las principales temáticas asociadas a los ejes conceptuales, con excepción del cuarto eje, referido a la investigación y educación, que se destaca con mayor detalle más adelante.

Tabla 4

Temáticas por eje conceptual

\begin{tabular}{lclc}
\hline \multicolumn{1}{c}{ Temáticas por eje conceptual } & Número de artículos & \multicolumn{1}{c}{ Temáticas por eje conceptual } & Número de artículos \\
\hline \multicolumn{1}{c}{ TA } & 1.938 & \multicolumn{1}{c}{ E0 } & 891 \\
\hline Gestión tecnológica & 294 & Nuevo Institucionalismo (NI) & 379 \\
Emprendimiento y empresarismo & 271 & $\begin{array}{l}\text { Aprendizaje y conocimiento } \\
\text { organizacional }\end{array}$ & 160 \\
Mercadeo & 255 & Cultura & 84 \\
Estrategia & 250 & Sociología organizacional & 65 \\
Finanzas & 207 & Teoría crítica y postmodernismo & 60 \\
$\begin{array}{l}\text { Ética y responsabilidad social } \\
\text { empresarial }\end{array}$ & 130 & Cambio organizacional & 37 \\
Administración pública & 120 & Gestión del conocimiento & 19 \\
Liderazgo & 87 & Nuevas formas de organización & 17
\end{tabular}




\begin{tabular}{|c|c|c|c|}
\hline Temáticas por eje conceptual & Número de artículos & Temáticas por eje conceptual & Número de artículos \\
\hline Contabilidad & 70 & Ecología de las organizaciones & 16 \\
\hline $\begin{array}{l}\text { Globalización e } \\
\text { internacionalización }\end{array}$ & 67 & $\begin{array}{l}\text { Lenguaje y comunicación } \\
\text { organizacional }\end{array}$ & 15 \\
\hline Gestión de la calidad & 40 & $\begin{array}{l}\text { Psicoanálisis en las } \\
\text { organizaciones }\end{array}$ & 14 \\
\hline PYMES & 39 & Critical Management Studies & 8 \\
\hline Operaciones y logística & 33 & $\begin{array}{l}\text { Teoría de la ambigüedad } \\
\text { organizativa }\end{array}$ & 6 \\
\hline Economía & 32 & Poder & 5 \\
\hline Reflexiones en torno a la TA & 16 & Reflexiones en torno a los E0 & 4 \\
\hline Consultoría & 12 & $\begin{array}{l}\text { Antropología de las } \\
\text { organizaciones }\end{array}$ & 2 \\
\hline Gestión de proyectos & 11 & & \\
\hline Gestión del fuero interno & 4 & & \\
\hline T0 & 221 & $\mathrm{I} \& \mathrm{E}$ & 160 \\
\hline Productividad y control & 59 & & \\
\hline Escuela de la contingencia & 50 & Educación & 81 \\
\hline Escuela de comportamiento & 32 & & \\
\hline Relaciones humanas & 26 & & \\
\hline Nuevas relaciones humanas & 25 & Investigación & 55 \\
\hline Burocracia & 16 & & \\
\hline Crisis & 7 & & \\
\hline Reflexiones en torno a la T0 & 5 & Pedagogía & 24 \\
\hline Moda & 1 & & \\
\hline
\end{tabular}

Fuente: elaboración propia.

En la TA. En primer lugar, los artículos sobre gestión tecnológica muestran trabajos relacionados con el uso de las herramientas tecnológicas para la gestión administrativa de las organizaciones a partir de las TIC, el diseño, la planificación y la transferencia. Al interior de las organizaciones se destaca el estudio de la complejidad organizacional producto de la incorporación de las nuevas tecnologías (Queiroz y de Vasconcelos, 2005), y la gestión tecnológica como consecuencia de la necesidad de desarrollar funciones de investigación y desarrollo (Hernández y Domínguez, 2008). Desde una mirada macro, se analizan las variables que influencian el descubrimiento y la difusión de las tecnologías en los mercados competitivos (Kimura, Moori y Asakura, 2005) y la gestión tecnológica como una elección estratégica de mercado ligada a la innovación (González-Ramos, Donate-Manzanares, Guadamillas-Gómez y Alegre-Vidal, 2014). 
En segundo lugar, la temática de emprendimiento y empresarismo enfatiza en los procesos de búsqueda de oportunidades, asunción de riesgos y ejecución de acciones para capitalizarlas y obtener beneficios, además de la creación y gestión del negocio. Autores como Cerdán-Chiscano, Jiménez-Zarco y Torrent-Sellens (2013), Hierro, Vasconcellos, Volpato y Laurindo (2013) y Castro, Galán y Bravo (2014) señalan la importancia de fortalecer las redes de emprendimiento para la interacción entre agentes y el éxito empresarial.

En tercer lugar, el tema del mercadeo da cuenta de actividades que ayudan al incremento del consumo, la búsqueda de nuevos clientes y el auge del mercado. En los textos revisados se destacan modelos y análisis de satisfacción de los consumidores para la mejora de la calidad, la estética de los productos, la diversión de los servicios, la generación de valor, etc. Algunos autores identificados en esta temática son: Cansado y Minciotti (2007), Ospina y Gil (2011) y Muhtaseb, Lakiotaki y Matsatsinis (2012).

3.2.1. EN LA TO. En primer lugar, se destaca el tema de la productividad y el control a partir del estudio o la práctica administrativa, $y$, más específicamente, en la mejora de los procesos organizacionales. Allí los textos detallan subtemas como la rendición de cuentas (0choa y Montes de 0ca, 2004; Sánchez, 2014), la eficiencia técnica (Guzmán y Montoya, 2011), la competencia laboral (Cequea y Núñez, 2011; Pineda-Zapata, Pérez-Ortega y Arango-Serna, 2012) y la gestión del control (Porporato y García, 2011).

En segundo lugar, la temática denominada escuela de la contingencia muestra relaciones entre la estructura de la organización y su contexto; entre algunos autores se destacan: Velásquez, Olaya y Franco (2009); Machado (2011), y Moreno y Reyes (2013). En la misma se subraya que no existe una única manera de administrar las organizaciones, debido a la dependencia del ambiente en el cual ellas se desarrollan.

En tercer lugar, se identifica la escuela del comportamiento, en la que se concibe a la organización como un sistema decisorio. Entre los subtemas relevantes se identificaron: toma de decisiones (Gomes, 2004; Goyal, Lu y Zhang, 2008; Santos y Barros, 2011), mecanismos de colaboración (Kay y Dyson, 2006; Pérez, 2008) y racionalidad limitada (Yen-Tsang, Csillag y Siegler, 2012).

3.2.2. EN LOS EO. En primer lugar, el tema del neoinstitucionalismo deja entrever la importancia de los estudios basados en el enfoque institucional, que concibe a las organizaciones como sistemas complejos gobernados por reglas y normas que logran un carácter legítimo y permanente y que buscan configurar un comportamiento estándar en 
los individuos. Allí se observan publicaciones relacionadas con subtemas como administración pública y gobernanza (Longo, 2010; Oliveira, Albuquerque y Pereira, 2012; Rótulo, Baudean, Abrucio y Cohn, 2014), isomorfismos y neoinstitucionalismo sociológico, político y económico (DiMaggio y Powell, 2005; Mangi, 2009; González, 2012), procesos y reglas institucionales (Cunha y Garzon, 2007; Machado-da-Silva y Vizeu, 2007), entre otros.

En segundo lugar, la temática de aprendizaje y conocimiento organizacional estudia cómo las organizaciones aprenden a partir de los individuos que la conforman; a su vez, dicho conocimiento se particulariza y de él se apropian los mismos individuos (Ortega, 2005; Benavides, 2011; Lucena y Cuhna, 2014).

En tercer lugar, el tema de la cultura se entendió como un conjunto de significados socialmente construidos dentro de la organización o manipulados por la administración. Entre los subtemas identificados se encuentran: cultura organizacional (Pérez, 2009; Leme, 2009; Alvarado y Monroy, 2013), cultura corporativa (Méndez, 2003; Martínez, 2010; Rodrigues y Sbragia, 2013), identidad organizacional (Carvajal, 2005; Arruda, 2007), imagen organizacional (Perozo y Montiel, 2008; Torres, Marinao y Chasco, 2012), interculturalidad (Hidalgo, Manzur, Olavarrieta y Farías, 2007; Álvarez, 2013), entre otros.

\subsection{Administración y organizaciones: conversaciones sobre investigación y educación}

En este apartado se retoman los artículos relacionados con el eje conceptual I\&E, con el fin de hacer énfasis y ampliar reflexiones en administración y organizaciones, así como brechas y retos de la investigación, educación y pedagogía en administración.

3.3.1. INVESTIGACIÓN. Este foco plantea perspectivas para el campo de la administración y las organizaciones, así como tensiones entre rigor, originalidad y relevancia investigativa. La cuestión del rigor hace énfasis en lo teórico y lo metodológico de acuerdo con las convenciones de una comunidad científica, mientras que la originalidad y la relevancia sugieren consideraciones necesarias para tal tipo de comunidad, al tener presente el impacto que se produce en el conocimiento para dirigir la aplicación práctica, replantear la comprensión de la realidad o legitimar aspectos en ella (Ofenhejm, Zambaldi y Alves de Moraes, 2011).

En el rigor investigativo desde la perspectiva teórica, se reconoce que el conocimiento científico es un proceso de acumulación que se apoya en un cuerpo pertinente establecido; 
allí se identifica el desafío de las reivindicaciones epistémicas como un acto mediante el cual los investigadores se involucran socialmente con el saber que movilizan, a partir de lo teórico, metodológico y performativo (Pina-Stranger, Sabaj, Toro y Matsuda, 2013). Por su parte, el rigor en el énfasis metodológico sugiere la aplicación de métodos e instrumentos acordes con la administración y las organizaciones; en los artículos relevados se muestra, por ejemplo, que la acción participante reconoce un auge del estudio de los fenómenos de carácter social relacionados con la gestión (Abib, Hoppen y Hayashi, 2013), los grupos focales permiten aprovechar las particularidades de indagación en el mundo virtual de los negocios (Houliez y Gamble, 2012; Safari, 2012) y el estudio de caso, a pesar de las críticas por su falta de cientificidad, se defiende como un método que permite diálogo entre los investigadores, las temáticas de análisis y la práctica (Mariotto, Pinto y Salati, 2014).

Respecto a la relevancia investigativa, se reconoce que el conocimiento es un bien público y la investigación una forma privilegiada de obtenerlo, que se legitima por su utilidad social, es decir, por los beneficios que genera para quienes hacen y aplican la gestión (Gutiérrez, Castaño y Vivares, 2013). No obstante, en las últimas décadas esa utilidad social hace referencia a la sociedad neoliberal, a lo que sirve al mercado; por ende, se ha indicado que la universidad debe tener un papel activo en alcanzar, "a través de grupos de investigación, soluciones para los problemas de la sociedad y nuevas alternativas de crecimiento, emprendimiento e innovación" (Higuita-López, Molano-Velandia y Rodríguez-Merchán, 2011, p. 209).

En relación con lo anterior, Netto y Bianchetti (2011), profesores adscritos a universidades brasileras, manifiestan que el sistema económico vigente y el impulso de los organismos internacionales (ej. Banco Mundial, Fondo Monetario Internacional, Organización Mundial del Comercio, Organización para la Cooperación y el Desarrollo Económico, etc.), han convertido el trabajo del investigador en un productivismo académico que necesita del conocimiento, incrementando así la precariedad de la investigación en 0ccidente. En esta misma línea, Sánchez-Torres y Rivera (2009), proponen medir las capacidades de investigación a partir del capital intelectual para las instituciones de educación superior, lo que evidencia una relación cada vez más pragmática entre investigación, calidad de la educación y gestión del conocimiento.

También en la relevancia y la originalidad de la producción científica, autores como Mascarenhas, Zambaldi y Alves de Moraes (2011) estudiaron la publicación científica en Brasil para denunciar el alejamiento de las particularidades socioculturales locales al tratar de 
imitar lo que se produce en Estados Unidos y el público especializado de investigadores que se encuentra distante de los gerentes, lo que revela tensiones que hay que replantear para que no se ignore lo disciplinar ni lo profesional.

Por último, se reconoce que uno de los mayores desafíos para el fortalecimiento de la investigación ha sido el surgimiento, el desarrollo y la consolidación de revistas académicas de alta calidad, insuficientes frente a la cantidad de grupos de investigación en administración (Malaver, 2006). Al respecto, un estudio reciente destaca que el fomento a la investigación en este campo requiere mayores recursos para investigar, estímulos económicos, retos en la política de ciencia y tecnología, comunidad científica y otras formas de divulgación de los resultados (Gutiérrez, Castaño y Vivares, 2013).

3.3.2. Educación. Este foco capta diversas problemáticas que se han venido discutiendo en las últimas décadas, como, por ejemplo, la reducción de la complejidad de los fenómenos estudiados en la administración (Mainardes y Domingues, 2010; Uribe, Ortiz y Domínguez, 2011), la americanización de la enseñanza y la instrumentalización del conocimiento (Alcadipani y Bertero, 2012), la eficiencia en educación como un factor primordial (Marcén y Martínez-Caraballo, 2012), las falencias en el relevo generacional (Hernández y Dueñas, 2005), la falta de adaptabilidad y uso de herramientas en la docencia virtual (Buil, Hernández, Sesé y Urquizu, 2012), el acoso laboral (bullying) y la violencia asociada con la docencia (Raya, Herruzo y Pino, 2009; León, 2009).

En el incremento de las críticas y cambios en la educación en administración se reconoce la fuerte influencia de los CMS, perspectiva europea que permite una visión diferente a la instrumental en el mundo de la gestión. En este sentido, se discute la forma de renovar la educación, los métodos de enseñanza, los vínculos entre la teoría y la práctica y el desarrollo crítico de los estudiantes. Respecto a las escuelas de negocios, se señala que la educación se ha convertido en un bien comercial (Calderón, 2004; Tognato, 2007; Guzmán, Restrepo y Trujillo, 2012), que los MBA, por ejemplo, son un producto estandarizado pero con un alto nivel simbólico, que implica estatus, prestigio y poder (Wood y Paes, 2004). Una crítica similar se establece con la calificación de los cursos de administración como fábricas y la tendencia oportunista en la formación de sus estudiantes (Pfeffer y Fong, 2003). En este sentido, la formación en administración está influyendo en el autointerés de los individuos con un impacto negativo en los valores, actitudes y comportamiento de sus alumnos (Fernández-Gago y Martínez-Campillo, 2012), catalogados como consumidores de la educación (Sánchez, Pintado, Talledo y Carcelén, 2009). 
A partir de las brechas que se identifican en la perspectiva educativa se cuestiona la disponibilidad limitada de los libros de calidad local para los cursos de administración (Wood y Paes, 2004), la alienación en la educación hacia el progreso económico (Calderón, 2004), el neoliberalismo como racionalidad para formar en administración (Hernández y Dueñas, 2005) y con ello la sensibilidad cada vez más apremiante de los estudiantes universitarios con la educación para el emprendimiento (Rodríguez y Prieto, 2009; Osorio y Pereira, 2011). En este sentido, se reconoce un modelo educativo que impulsa el productivismo académico y una formación para el empleo (Uribe, Ortiz y Domínguez, 2011) que desarrolla el ejercicio profesionalizante del conocimiento, dejando de lado el cultivo del saber por sí mismo (Miranda, 2005).

En este sentido, los retos de dicho enfoque aluden a cambios en la educación en administración (Friga, Bettis y Sullivan, 2004). Se propone renovarla a partir de los contenidos, métodos de enseñanza, vínculos entre teoría y práctica y desarrollo de la crítica y analítica en los estudiantes (Wood y Paes, 2004); incluso, se recomienda tener presente que desde la formación en la escuela hay que potenciar la educación superior (Velásquez, Novoa y Mayorga, 2006; Aguilar, 2009). La universidad como organización debe estar conectada con los sistemas de interés (sociedad civil, gobierno, medios y publicaciones, contexto, etc.) (Duque, 2009) para propiciar el desarrollo de habilidades, no solo técnicas, sino capacidad oral y escrita, trabajo en equipo, búsqueda de información, manejo de aplicaciones informáticas, de análisis, síntesis y crítica (Lobo, Escobar y Arquero, 2009).

A partir de los escándalos financieros de la década de 2000, algunos autores plantean que la administración debe enseñarse teniendo presente el respeto por el medio ambiente y los intereses por el largo plazo (Wood y Paes, 2004). Otros reflexionan sobre la necesidad del desarrollo de una perspectiva más amplia y más realista con respecto a los valores de los individuos (Lourenço y Knop, 2011), una gerencia que se sustente en pilares axiológicos, praxiológicos, epistemológicos y teleológicos de acuerdo con la que se redimensionen los estilos de dirección a partir de la colaboración y la sensibilidad con los procesos de transformación social (Rondón, 2011).

3.3.3. Pedacocía. Este foco capta la interacción que poseen la educación y la investigación a partir de la enseñanza de la administración en un ámbito organizacional. En la década de 1990 surgió el llamado "optimismo pedagógico", que buscaba un conjunto de reformas para planificar la educación en los planos económico y social, pues se reconocía la inoperancia de los sistemas educativos con una autonomía socavada en la burocratización y carente de desafíos (Aguilar, 2009). Allí los proyectos educativos y curriculares se 
quedaron en una concreción institucional, un instrumento descontextualizado ausente de las condiciones idóneas para facilitar el aprendizaje de las partes.

En la actualidad la enseñanza tiene el reto de proveer respuestas a la complejidad del campo de estudio en la posmodernidad y las nuevas circunstancias generadas por la sociedad del conocimiento (Blanco-Encomienda y Latorre-Medina, 2012), la revolución tecnológica y la reorganización de la economía a escala mundial que obliga a crear ventajas comparativas, incluso, en la educación (Santana, 2000). En consecuencia, algunas investigaciones señalan la existencia de un discurso institucional que demuestra la intencionalidad de formar en competencias en el ámbito de la administración; no obstante, en la realidad los cursos no tienen tal orientación sistemática y articulada entre los elementos que integran el proceso de enseñanza-aprendizaje (Nunes y Patrus-Pena, 2011). Existe así un desafío latente de una pedagogía que se adapte a las características del escenario social, con un proyecto pedagógico que no se quede en el papel sino que se integre en la práctica de la docencia universitaria.

En la enseñanza de la administración, las limitaciones son mucho más que la preocupación por la didáctica; por lo tanto, la reflexión pedagógica debe trascender la consideración metodológica y vislumbrar que “la enseñanza se acople en sincronía con el avance del saber tanto en lo científico como en lo tecnológico, y se procure un equilibrio entre lo teórico y lo pragmático, entre el desarrollo de competencias tanto cognitivas como técnicas" (Marín, 2005, p. 45). En este sentido, se plantea la posibilidad de una pedagogía crítica no solo a partir de los contenidos curriculares, sino del estímulo de la posición crítica de estudiantes en administración que han estado acostumbrados a buscar recetas y fórmulas mágicas en la gestión (Paes y Rodrigues, 2006).

Entre los retos a los que se enfrenta la pedagogía se resalta la generación de espacios de diálogos constructivos "en los cuales el docente, con criterio tolerante y sensato, reconoce la mente del otro (estudiante) y propicia la adopción de competencias mediante manifestaciones hermenéuticas - explicativas - prescriptivas, soportadas en diferentes acciones metódicas y factuales" (Marín, 2005, p. 56). Se requiere un esfuerzo muy amplio por parte de profesores y estudiantes, así como un manejo de la coherencia entre los contenidos de los cursos y sus métodos de transmisión, además de una buena sustentación al interior del sistema educativo tradicional y un apoyo institucional muy fuerte (Aguilar, 2009). Estos cambios en la enseñanza del campo administrativo y organizacional implican modificaciones de la política educativa y de la mentalidad de directivos, docentes y estudiantes. 


\section{Discusión}

La administración y la organización configuran un diálogo de mutua implicancia, ya que bien se podría afirmar que no existe una organización que no sea susceptible de ser administrada. Esta idea, no sólo expresa la íntima relación que existe entre una y otra, sino también, la necesidad de profundizar y comprender las limitaciones y alcances de cada una de ellas. Esto es lo que justamente posibilita la emergencia de diversas conversaciones sobre las perspectivas teóricas, temáticas, autores, redes de publicación e instituciones que desarrollan conocimiento sobre estos dos campos de saberes.

Aunado a esto, hay autores que forman parte de esta conversación, que no sólo están inscritos en una perspectiva particular, sino que también desarrollan una serie de temas y provienen de diversas latitudes. Es más, son ellos los que propician, mantienen y avigoran los diálogos. Los resultados de la investigación exponen al mundo académico un marco de clasificación que permite esclarecer los temas, identificar la institución y posturas teóricas que los investigadores han desarrollado en los últimos 15 años respecto a la administración y las organizaciones, así como en la investigación y la educación. Estos aportes representan una primera aproximación, que a modo de punto de partida, permitirá desarrollos posteriores en Latinoamérica sobre las conversaciones que aquí se presentan.

La propuesta de clasificación teórica-metodológica, aún inacabada, constituye en sí misma un aporte de la investigación. Si bien esta no está exenta de debates y controversias, favorece la discusión y promueve el estudio de las organizaciones como campo de interés investigativo. Recalcar a su vez, que la TA, la T0 y los E0 se desarrollan paralelamente, y en ese sentido, no es que los EO expresen una evolución "mejorada" de la TA y de la T0, y mucho menos que muestren su caducidad. Por el contrario, los E0 son una aproximación alternativa al estudio de las organizaciones y para ello retoman la TA y la T0 de una manera crítica y comprensiva. Entonces, estos ejes no sólo están vigentes, sino que son de creciente interés por parte de los investigadores en el campo de la administración y las organizaciones. Es decir, que el estudio de las organizaciones tiene una connotación de no exclusión desde el punto de vista teórico-conceptual, más allá de que en Latinoamérica existe una fuerte inclinación hacia la TA.

Otra conversación emergente se relaciona con los temas asociados a cada uno de los ejes conceptuales propuestos. Es propio del ámbito administrativo encontrar una diversidad de temas vinculados con la gestión y la intervención organizacional, de hecho, es una de las razones, entre otras, por las cuales la administración se ha vuelto tan preciada 
y requerida, gracias a su polivalencia y posibilidades de aplicación en las empresas. No obstante, los resultados de la investigación muestran que los ejes de la TA y la T0 adolecen de una mirada comprensiva, tanto de las acciones que propician al interior de las organizaciones, como de las implicaciones éticas y humanas que conllevan dichas acciones. Esto difiere de los ejes EO e I\&E, en donde no solo se evidencia una menor cantidad de artículos publicados, sino que las aproximaciones tienden a ser más comprensivas que aplicativas.

Con esto no se busca propiciar una posición epistemológica caracterizada por una polaridad irreconciliable de perspectivas, la funcional-positivista por un lado, y la comprensiva-crítica por el otro; ni tampoco estigmatizar valorativamente alguna de ellas. El estudio organizacional implica reconocer la realidad de las organizaciones en su singularidad, así como apreciar que su operatividad se ve regida por las lógicas de la eficiencia y la productividad. Por ello, lejos de defender la idea de tomar posición por alguna perspectiva teórica en particular, se busca fomentar la complementariedad y la conversación entre ellas.

Como se evidencia en la tabla 4, muchas de las temáticas se podrían clasificar dentro de diversas áreas de conocimiento, y no solo en la administración, como por ejemplo: economía, gestión humana, finanzas, contabilidad, mercadeo, derecho, logística y operaciones, entre otras. Tal como se observa hoy en día en las carreras profesionales que se ofertan en universidades, dichas áreas han migrado a disciplinas independientes que forman sus propios profesionales en campos de conocimiento específicos. Entonces, ¿qué le queda a la administración como disciplina? En otras palabras, ¿cuál es la naturaleza de la administración y cómo se define su identidad en tanto campo de saber y en tanto práctica, si paulatinamente queda despojada de las áreas de conocimiento que hasta hoy le han dado solidez teórica?

Por otro lado, conversaciones desde el punto de vista de la investigación, resaltan la gran influencia del pensamiento neoliberal, que promueve en los investigadores una preocupación por la publicación y no tanto por la relevancia y la originalidad, lo cual fomenta un pragmatismo investigativo (Sánchez-Torres y Rivera, 2009). Así, antes de centrar el interés en la formulación del problema a ser investigado, se pone atención en las posibilidades de publicación 0 , incluso, se adapta la investigación para lograr tal objetivo. Esta situación puede configurar una traslación de medios hacia fines, en la que las lógicas de la racionalidad instrumental influencian la investigación, al intentar convertirla en un productivismo académico que capitalice el conocimiento (Netto y Bianchetti, 2011). 
En cuanto a la educación, es importante subrayar el papel que tiene la formación teórica como sustento firme y sólido para abordar críticamente los fenómenos organizacionales. Esto, a su vez, permite la construcción de criterio y la posibilidad de comprender la realidad organizacional, así como las consecuencias de las acciones de la gestión en la intervención; que de lograrse, se generaría un contraste con la instrumentalización del conocimiento (Alcadipani y Bertero, 2012). Pero la educación en administración, a partir de las tecnologías educativas y las didácticas con las que orientan la formación de los profesionales en espacios de aprendizaje lúdicos, flexibles, amenos, y ausentes de frustración, termina por minimizar la incertidumbre con el fin de garantizar los logros del aprendizaje. Concebida así la educación, ¿qué le queda a ella para la formación de ciudadanos? ¿Acaso ésta, sustraída de la complejidad de la vida, forma sujetos para la transformación de las sociedades contemporáneas? Máxime que la sociedad de hoy se entiende como una sociedad de organizaciones gobernadas en mucho por administradores.

Finalmente, es importante reflexionar sobre la presencia de otros marcos teóricos que tengan un enfoque más comprensivo y que hagan uso de los aportes de las ciencias sociales y humanas para analizar las realidades organizacionales latinoamericanas. Como se ya se evidenció, la región presenta una gran inclinación hacia una miranda funcional-pragmática estadounidense. En ese sentido, los EO representan una oportunidad para propiciar otro tipo de conversaciones caracterizadas por el abordaje de los problemas organizacionales desde una mirada crítica. Si bien este campo es conocido y desarrollado en países como México y Brasil, en el resto de Latinoamérica aún están en proceso de divulgación y reconocimiento.

\section{Limitaciones y líneas de investigación futuras}

Una primera limitación es que debido al objetivo y a la orientación del estudio realizado, se optó por hacer una descripción más holística de los resultados para profundizar en aquellos referidos a la investigación y la educación en administración y organizaciones. A esto se le suma la clasificación de las revistas en los cuatro ejes temáticos descritos. Si bien dicha sistematización se fundamentó teóricamente, la decisión de clasificación de los artículos depende de un proceso de interpretación de los investigadores. En ese sentido, la propuesta que aquí se presenta es una posibilidad entre muchas otras, que no está exenta de controversias y discusiones. Al ser este trabajo un punto de partida, el análisis de las conversaciones han sido acotadas a los objetivos planteados y no se evidencia, en esta primera etapa, una relación dialógica que logre identificar la interacción académica entre los autores bajo una temática, campo o perspectiva teórica particular. 
La investigación identificó un panorama de la realidad del campo administrativo y organizacional en la literatura especializada en Latinoamérica, que otorga a los futuros investigadores posibilidades de conocer, ubicarse y construir proyectos de investigación en dichos ámbitos. De esta manera, se podrían considerar algunas líneas de investigación que permitan dar continuidad a este trabajo. La primera, referida a la identificación de diversas conversaciones que expresen la inquietud académica de los investigadores en Latinoamérica, y con ello posibilitar el reconocimiento de las conceptualizaciones y aproximaciones generadas sobre la administración y el estudio de las organizaciones, además de la interacción entre los artículos. La segunda, direccionada a los marcos teóricos y metodológicos de los E0, que podrían llegar a complementar la formación de los administradores: ¿Cuál es la incidencia de los E0 en la investigación en América Latina? ¿Qué nuevos marcos de conocimiento ofrecen los E0 para abordar las problemáticas organizacionales y las relaciones que esto genera con la administración? Y la tercera, relacionada con el papel que cumple la educación en administración (como pueden ser las maestrías y los doctorados) en la formación de futuros investigadores desde una perspectiva crítica y comprensiva: ¿Cómo integrar la propuesta teórica y metodológica de los E0 en la formación de administradores? ¿Qué perfil de administrador se está formando actualmente?

La realidad en el campo de la administración y las organizaciones es diversa y por lo mismo brinda espacios para pensar las prácticas administrativas en las organizaciones desde distintas ópticas y métodos. Esta heterogeneidad invita a reflexionar aún más sobre los fundamentos de la administración y las implicaciones de sus intervenciones en las organizaciones, y sobre las conversaciones que se han de propiciar a partir de los artículos para el avance del conocimiento. Asimismo, conlleva también a efectuar una autocrítica, cuyas repercusiones permitan no solo un mejor desarrollo docente e investigativo en las instituciones académicas y en el quehacer de los profesores e investigadores, sino en el modelo de ciudadano y con ello de sociedad, que se construye desde la perspectiva actual de dicho campo de saber.

\section{Referencias}

Abib, G.; N. Hoppen e P. Hayashi. (2013). Observação participante em estudos de administração da informação no Brasil. RAE, 53 (6), 604-616.

Aguilar, L. (2009). Políticas del cambio en educación y gestión de la innovación. Innovar, 19, 9-24. Ailon, G. (2006). What B would otherwise do: A critique of conceptualizations of "power" in organizational theory. Organizations, 13 (6), 771-800. 
Aktouf, 0. (1998). La administración: entre tradición y renovación, $3^{\text {a }}$ ed. Cali: Artes Gráficas del Valle.

Alcadipani, R. e C. Bertero. (2012). Guerra fria e ensino do management no Brasil: o caso da FGV-EAESP. RAE, 52 (3), 284-299.

Alvarado, Ó. y R. Monroy. (2013). Cultura organizacional en una empresa propiedad de sus trabajadores. Cuadernos de Administración, 26 (47), 259-283.

Álvarez, L. (2013). Interculturalidad: inclusión y exclusión en la política de gestión de la diversidad en Barcelona. Revista del CLAD, 57, 123-156.

Arruda, I. (2007). Construção e desconstrução de fronteiras e identidades organizacionais: história e desafios do Mcdonald's. RAE, 47 (1), 60-70.

Augier, M.; J. March, and B. Ni Sullivan. (2005), Notes on the evolution of a research community: organization studies in anglophone North America, 1945-2000, Organization Science, $16(1), 85-95$.

Barba, A. (2013). Administración, teoría de la organización y estudios organizacionales. Tres campos de conocimiento, tres identidades. Gestión y Estrategia, 44 (2), 139-151.

Batista-dos-Santos, A.; J. Mata de Lima e L. Holanda. (2010). Epistemologia e metodologia para as pesquisas críticas em administração: leituras aproximadas de Horkheimer e Adorno. $R A E, 50$ (3), 312-324.

Bédard, R. (2003). Los fundamentos del pensamiento y las prácticas administrativas. El rombo y las cuatro dimensiones filosóficas. Ad-Minister, 3, 68-87.

Bédard, R. (2004). Los fundamentos del pensamiento y las prácticas administrativas: la trilogía administrativa. Ad-Minister, 4, 80-108.

Benavides, M. (2011). El conocimiento como recurso estratégico para el aprendizaje interorganizativo. Revista Venezolana de Gerencia, 16 (56), 564-577.

Betancourt, G. (2003). De la historia empresarial a la historia organizacional. Innovar, 13 (22), 199-210.

Blanco-Encomienda, F. y M. Latorre-Medina. (2012). La enseñanza práctica y pre-profesional en el marco de las ciencias administrativas. Innovar, 22 (45), 69-82.

Buil, I.; B. Hernández, F. Sesé y P. Urquizu. (2012). Los foros de discusión y sus beneficios en la docencia virtual: recomendaciones para un uso eficiente. Innovar, 22 (43), 131-143.

Burns, T., and G. Stalker. (1961). The management of innovation. Oxford, UK: Oxford University Press.

Calderón, A. (2004). Repesando o papel da universidade. RAE, 44 (2), 104-108.

Calderón, H. y D. Castaño. (2005). Introducción. En H. G. Calderón y D. G. Castaño (Comps.), Investigación en administración en América Latina: evolución y resultados (pp. 7-12). Manizales: Universidad Nacional de Colombia. 
Cansado, T. e S. Minciotti. (2007). Serviço de atendimento ao cliente terceirizado ou próprio? Uma análise da satisfação do cliente usuário. RBGN, 9 (25), 81-95.

Carvajal, J. (2005). El problema de la existencia y la identidad de las organizaciones. Innovar, 15 (25), 25-35.

Castro, I.; J. Galán y S. Bravo. (2014). Emprendimiento y capital social: evidencia de una incubadora empresarial colombiana. Innovar, 24, 91-100.

Cequea, M. y M. Núñez. (2011). Factores humanos y su influencia en la productividad. Revista Venezolana de Gerencia, 16 (53), 116-137.

Cerdán-Chiscano, M.; A. Jiménez-Zarco y J. Torrent-Sellens. (2013). Valorando los programas de apoyo al emprendedor en las incubadoras de empresas en Cataluña. Revista Venezolana de Gerencia, 18 (63), 408-433.

Chia, R., and R. Holt. (2008). The nature of knowledge in business schools. Academy of Management Learning \& Education, 7 (4), 471-486.

Clegg, S. (1990). Modern organizations. Organization studies in the postmodern world. London: SAGE.

Clegg, S. R., and C. Hardy. (1996). Introduction. Organizations, organization and organizing. In S. Clegg, C. Hardy, and W. Nord (Eds.), Handbook of Organization Studies (pp. 1-28). London: Sage.

Clegg, S., and J. Bailey. (2008). Introduccion. In S. Clegg, and J. Bailey (Eds.), International encyclopedia of organization studies (pp. xiii-xviii). Los Angeles: Sage.

Clegg, S., and D. Dunkerley. (1977). Critical issues in organisations. London: Routledge and Kegan Paul.

Contreras, J. y M. Crespo. (2005). ¿Hacia un nuevo sentido de la gerencia? Revista Venezolana de Gerencia, 10 (32), 583-604.

Cunha, R., and F. Garzon. (2007). Performance-based potable water and sewer service regulation: The regulatory model. Cuadernos de Administración, 20 (34), 283-298.

Daniels, J. (1999). La enseñanza, la investigación y el servicio a la comunidad. Academia. Revista Latinoamericana de Administración, 23, 28-34.

Dávila, C. (2001). Teorías organizacionales y administración. Bogotá: McGraw-Hill.

Dávila, C. (2005). Generación de conocimiento administrativo en América Latina: ¿Realidad, necesidad o utopía? En H. Calderón y D. Castaño (Comps.), Investigación en Administración en América Latina: evolución y resultados (pp. 39-43). Manizales: Universidad Nacional de Colombia.

De la Rosa, A. (2012). Seminario de Teoría de la Organizaciones. Memorias de clase. Medellín: Universidad EAFIT.

De Mattos, P. (2009). Administração é ciência ou arte? 0 que podemos aprender com este mal-entendido? RAE, 49 (3), 349-360. 
DiMaggio, P. e W. Powell. (2005). A gaiola de ferro revisitada: isomorfismo institucional e racionalidade coletiva nos campos organizacionais. $R A E, 45$ (2), 74-90.

Donaldson, L. (2003). Position statement for positivism. En R. Westwood, and S. Clegg (Edits.), Debating Organization (pp. 116-127). 0xford: Blackwell.

Duque, C. y M. Royuela. (2005). La investigación como motor de desarrollo. En H. Calderón y D. Castaño (Comps.), Investigación en Administración en América Latina: evolución y resultados (pp. 15-34). Manizales: Universidad Nacional de Colombia.

Duque, E. (2009). La gestión de la universidad como elemento básico del sistema universitario: una reflexión desde la perspectiva de los stakeholders. Innovar, 19, 25-41.

Fayol, H. (2003). Administración industrial y general. Bogotá: Adigrama.

Fernández-Gago, R., and A. Martínez-Campillo. (2012). Teaching business management from a perspective beyond self-interest. Innovar, 22 (46), 165-174.

Friga, P.; R. Bettis e R. Sullivan. (2004). Mudanças no ensino em administração: novas estratégias para o século XXI. RAE, 44 (1), 96-115.

Gardfield, E. (1972). Citation analysis as a tool in journal evaluation. Science, 178 (60), 471-479.

Gomes, R. (2004). Who are the relevants stakeholders to the local government context? Empirical evidences on environmental influences in the decision-making process of english local authorities. BAR, 1 (1), 34-52.

Gonzales-Miranda, D. (2014). Los estudios organizacionales. Un campo de conocimiento comprensivo para el estudio de las organizaciones. Innovar, 24 (54), 43-58.

Gonzales-Miranda, D. y M. Gentilin. (2012). Panorama del estudio de las organizaciones. Una caracterización del campo organizacional con base en tres ejes temáticos, 2000-2011. Análisis Organizacional, 1 (5), 1-28.

González, J. (2012). Reingeniería de procesos de negocio (BPR): análisis de un caso desde la perspectiva del nuevo institucionalismo sociológico. Innovar, 22 (46), 129-148.

González-Ramos, M.; M. Donate-Manzanares, F. Guadamillas-Gómez y J. Alegre-Vidal. (2014). Relación entre el dinamismo percibido, la postura tecnológica y los resultados de innovación. Journal of Technology Management \& Innovation, 9 (2), 131-144.

Goyal, M.; J. Lu, and G. Zhang. (2008). Decision making in multi-issue e-market auction using fuzzy techniques and negotiable attitudes. Journal of Theoretical and Applied Electronic Commerce Research, 3 (2), 97-110.

Gutiérrez, L.; G. Castaño y J. Vivares. (2013). Estímulos y restricciones para la investigación en administración en Colombia. Innovar, 23 (49), 5-16.

Guzmán, A.; J. Restrepo y M. Trujillo. (2012). Instituciones de educación superior con ánimo de lucro en Colombia. Innovar, 22 (43), 67-76.

Guzmán, I. y J. Montoya. (2011). Eficiencia técnica y cambio productivo en el sector ferroviario español de vía ancha. Innovar, 21 (40), 219-234. 
Hancké, B. (2009). Intelligent research design. A guide for beginning researchers in the social sciences. Oxford, UK: 0xford University Press.

Hatch, M. J. (1997). Organization theory. Modern, symbolic and postmodern perspectives. Oxford, UK: Oxford University Press.

Hernández, A.; J. Saavedra y M. Sanabria. (2007). Hacia la construcción del objeto de estudio de la administración: una visión desde la complejidad. Investigación y Reflexión, 15 (1), 91-112.

Hernández, C. y Ó. Domínguez. (2008). Perspectivas y consideraciones sobre el aporte biológico al desarrollo de la gestión de tecnología. Innovar, 18 (32), 47-64.

Hernández, I. y M. Dueñas. (2005). Contribución al debate sobre la educación y el mercado laboral en la administración pública en Colombia. Innovar, 15 (26), 13-32.

Hidalgo, P.; E. Manzur, S. Olavarrieta y P. Farías. (2007). Cuantificación de las distancias culturales entre países: un análisis de Latinoamérica. Cuadernos de Administración, 20 (33), 253-272.

Hierro, S.; E. Vasconcellos, M. Volpato and A. Laurindo. (2013). Barriers and facilitators of collaborative management in technological innovation projects. Journal of Technology Management \& Innovation, 8, 151-164.

Higuita-López, D.; J. Molano-Velandia y M. Rodríguez-Merchán. (2011). Competencias necesarias en los grupos de investigación de la Universidad Nacional de Colombia que generan desarrollos de base tecnológica. Innovar, 21 (41), 209-224.

Horkheimer, M. (2002). Crítica de la razón instrumental. Madrid: Trotta.

Houliez, C., and E. Gamble. (2012). Augmented focus groups: On leveraging the peculiarities of online virtual worlds when conducting in-world focus groups. Journal of Theoretical \& Applied Electronic Commerce Research, 7 (2), 31-51.

Ibarra, E. (2006). Teoría de la organización. Mapa conceptual de un territorio en disputa. En E. De la Garza (Ed.), Tratado latinoamericano de sociología de la empresa, México: Fondo de Cultura Económica.

Ibarra, E. y L. Montaño. (1990). Teoría de la organización: desarrollo histórico, debate actual y perspectivas. En E. Ibarra y L. Montaño (Ed.), Teoría de la organización: fundamentos y controversias (pp. vii-xxvi). México: UAM-I.

Ibarra, E. y L. Montaño. (1992). Mito y poder en las organizaciones. México: Trillas.

Jurado, J. C. (2015). Hacia una arqueología de la administración. Cuadernos de Administración, 28 (50), 113-136.

Kay, R., and L. Dyson. (2006). Learning to collaborate and collaborating to learn: An experiential approach to teaching collaborative systems. Journal of Theoretical \& Applied Electronic Commerce Research, 1 (2), 36-44.

Kimura, H.; R. Moori e 0. Asakura. (2005). Análise da difusão tecnológica usando algoritmos genéticos. Revista de Administração de Empresas, 45 (3), 25-39. 
Lawrence, P. y W. Lorsh. (1973). Organización y ambiente. Barcelona: Labor.

Leme, M. (2009). Organizational culture and the the renewal of competences. BAR, 6 (1), 1-14.

León, E. (2009). Angustia docente: una revisión de la investigación del malestar y la violencia docente en Latinoamérica. Innovar, 19, 91-110.

Lobo, A.; B. Escobar, and J. Arquero. (2009). Using empirical based case studies to improve motivation, non-technical skills and content learning: A longitudinal study of an EHEA experience. Innovar, 19, 43-52.

Longo, F. (2010). Ejes vertebradores de la gobernanza en los sistemas públicos. Un marco de análisis en clave latinoamericana. Revista del CLAD, 46, 73-102.

López, F. (1999). La administración como sistema gnoseológico. En búsqueda de un objeto de estudio. Revista Universidad EAFIT, 113, 19-40.

Lourenço, C. e M. Knop. (2011). Ensino Superior em Administração Percepção da Qualidade deServiços: uma aplicação da escala SERVQUAL. RBGN, 13 (39), 219-233.

Lucena, E., and C. Cuhna. (2014). Retail executives' professional learning contents. RBGN, 16 (50), 43-59.

Machado, M. (2011). Variáveis contingenciais aos métodos de valoração dos produtos: estudo empírico em PME's industriais portuguesas. RBGN, 13 (41), 394-414.

Machado-da-Silva, C. e F. Vizeu. (2007). Análise institucional de práticas formais de estratégia. $R A E, 47$ (4), 89-100.

Mainardes, E. e M. Domingues. (2010). Qualidade de cursos de administração e instituições de ensino superior em Joinville - SC: um estudo sobre fatores relacionados ao mercado de trabalho na percepção dos alunos. RBGN, 12 (35), 208-223.

Malaver, F. (1999). La investigación en gestión empresarial. Academia. Revista Latinoamericana de Administración, 23, 62-77.

Malaver, F. (2006). El despegue de la investigación colombiana en administración: análisis de sus avances en el periodo 2000-2006. Cuadernos de Administración, 19 (32), 71-109.

Mangi, L. (2009). Neoinstitutionalism and the appropriation of bourdieu's work: A critical assessment. RAE, 49 (3), 323-336.

Marcén, M. y N. Martínez-Caraballo. (2012). Gestión eficiente del tiempo de los universitarios: evidencias para estudiantes de primer curso de la Universidad de Zaragoza. Innovar, 22 (43), 117-130.

Marín, D. A. (2005). La enseñanza de las teorías de la administración: limitantes epistémicos y posibilidades pedagógicas. Innovar, 15 (26), 43-58.

Marín-Idárraga, D. A. (2007). Administración y racionalidad. Sotavento, 14, 8-41.

Marín-Idárraga, D. A. (2012). Consideraciones epistemológicas en torno al carácter científico de la administración. Innovar, 22 (16), 39-52. 
Mariotto, F.; P. Pinto, and G. Salati. (2014). What is the use of a single-case study in management research? $R A E, 54$ (4), 358-369.

Martínez, M. (2010). Relaciones entre cultura y desempeño organizacional en una muestra de empresas colombianas: reflexiones sobre la utilización del modelo de Denison. Cuadernos de Administración, 23 (40), 163-190.

Martins, A., e D. Barrera. (2012). A hermenêutica no campo organizacional: duas possibilidades interpretativistas de pesquisa. RBGN, 14 (44), 261-273.

Mascarenhas, A.; F. Zambaldi, e E. Alves de Moraes. (2011). Rigor, relevância e desafios da academia em Administração: tensões entre pesquisa e formação profissional. RAE, 51 (3), 265-279.

Mayo, E. (1972). Problemas humanos de una civilización industrial. Buenos Aires: Nueva Visión.

Méndez, C. (2003). Metodología para describir la cultura corporativa: estudio de caso en una empresa colombiana del sector industrial. Cuadernos de Administración, 16 (25), 139-171.

Miranda, N. (2005). Las instituciones educativas de nivel superior en México: posicionamiento y preferencias de los estudiantes en torno a la oferta educativa de la zona Tampico, Madero y Altamira. Innovar, 19, 33-45.

Montaño, L. (1994). Modernidad, postmodernismo y organización. Una reflexión acerca de la noción de estructura postburocrática. En L. Montaño (Ed.), Argumentos para un debate sobre la modernidad. Aspectos organizacionales y económicos (pp. 67-91). México: UAM-I.

Montaño, L. (2004). El estudio de las organizaciones en México, una perspectiva social. En L. Montaño (Ed.), Los estudios organizacionales en México. Cambio, poder, conocimiento e identidad (pp. 9-39). México: UAM-I, Universidad de Occidente y Miguel Ángel Porrúa.

Moreno, C. y J. Reyes. (2013). El valor de la estrategia ambiental proactiva: una evaluación empírica del enfoque contingente de las capacidades dinámicas. Cuadernos de Administración, 26 (47), 87-118.

Muhtaseb, R.; K. Lakiotaki, and N. Matsatsinis. (2012). Applying a multicriteria satisfaction analysis approach based on user preferences to rank usability attributes in e-tourism websites. Journal of Theoretical \& Applied Electronic Commerce Research , 7 (3), 28-48.

Muñoz, R. (2011). Formar en administración. Por una nueva fundamentación filosófica. Medellín: Fondo Editorial Universidad EAFIT.

Netto, A. e L. Bianchetti. (2011). (Des)fetichização do produtivismo acadêmico: desafios para o trabalhador-pesquisador. RAE, 51 (3), 244-254.

Nunes, S. e R. Patrus-Pena. (2011). A pedagogia das competências em um curso de administração: o desafio de passar do projeto pedagógico à prática docente. $R B G N, 13$ (40), 281-299.

Ochoa, H., y Y. Montes de 0ca. (2004). Rendición de cuentas en la gestión pública: reflexiones teóricas. Revista Venezolana de Gerencia, 9 (27), 455-472.

Ofenhejm, A.; F. Zambaldi e E. Alves de Moraes. (2011). Rigor, relevância e desafios da academia em administração: tensões entre pesquisa e formação profissional. RAE, 51 (3), 265-279. 
Oliveira, J.; A. Albuquerque, and R. Pereira. (2012). Governance, succession, and formalization of management in family businesses: (Re)organizing the role played by multi-generation families. RBGN, 43 (14), 176-192.

Ortega, R. (2005). Aprendizaje y acumulación de capacidades tecnológicas en un grupo del sector siderúrgico. Innovar, 15 (25), 90-102.

Osorio, F. y F. Pereira. (2011). Hacia un modelo de educación para el emprendimiento: una mirada desde la teoría social cognitiva. Cuadernos de Administración, 24 (43), 13-33.

Ospina, S. y I. Gil. (2011). Índices nacionales de satisfacción del consumidor. Una propuesta de revisión de la literatura. Cuadernos de Administración, 24 (43), 35-57.

Ottewill, R. (2003). What's wrong with instrumental learning? The case of business and management. Education + Training, 45 (4), 189-196.

Paes, A. e M. Rodrigues. (2006). Pedagogia crítica no ensino da administração: desafios e possibilidade. RAE, 46, 10-22.

Peci, A. (2003). Estrutura e ação nas organizações: algumas perspectivas sociológicas. RAE, 43 (1), 24-35.

Pérez, A. (2009). Cultura organizacional: algunas reflexiones a la luz de los nuevos retos. Revista Venezolana de Gerencia, 14 (46), 183-194.

Pérez, M. (2008). La cooperación empresarial en comunidades rurales ¿mecanismo del desarrollo endógeno? Revista Venezolana de Gerencia, 13 (41), 9-29.

Perozo, G. y Montiel, L. (2008). Imagen corporativa del sistema de franquicia en el sector de juegos de loterías. Revista Venezolana de Gerencia, 13 (42), 198-219.

Pfeffer, J. e C. Fong. (2003). 0 fim das escolas de negócio? RAE, 43 (2), 11-28.

Pina-Stranger, Á.; 0. Sabaj, P. Toro y K. Matsuda. (2013). Estrategias académicas de inserción científica: una propuesta metodológica para el estudio de las reivindicaciones epistémicas en los artículos de investigación. Innovar, 23 (48), 67-82.

Pineda-Zapata, U.; G. Pérez-Ortega y M. Arango-Serna. (2012). Medición del impacto de las competencias laborales en la productividad de los procesos: caso de una empress manufacturera. Innovar, 22 (45), 37-50.

Podestá, P. y J. Jurado. (2003). Fundamentos del saber administrativo. Cuadernos de Difusión, 15, 81-100.

Porporato, M. y N. García. (2011). Sistemas de control de gestión: un estudio exploratorio de su efecto sobre el desempeño. Academia. Revista Latinoamericana de Administración, 47, 61-77.

Pugh, D. (1997). Organization Theory. London: Penguin Books.

Queiroz, A. e F. de Vasconcelos. (2005). Organizações, Confiabilidade e Tecnologia. Revista de Administração de Empresas, 45 (3), 40-51. 
Ramírez, G.; G. Vargas y A. De la Rosa. (2011). Estudios organizacionales y administración. Constrastes y complementariedades: caminando hacia el eslabón perdido. Forum Doctoral, 3 (3), 7-54.

Raya, A.; J. Herruzo, and M. Pino. (2009). Predictors for bullying at work in the field of education. Innovar, 19, 65-71.

Reed, M. (1996). Organizations theorizing: A historically contested terrain. In S. Clegg, C. Hardy, and W. Nord. Handbook of Organization Studies (pp. 31-56). London: Sage.

Rodrigues, I., and R. Sbragia. (2013). The cultural challenges of managing global project teams: a study of brazilian multinationals. Journal of Technology Management \& Innovation, 8, 38-52.

Rodríguez, C. y F. Prieto. (2009). La sensibilidad al emprendimiento en los estudiantes universitarios. Estudio comparativo Colombia-Francia. Innovar, 19, 73-89.

Rondón, Y. (2011). Categorías de la acción gerencial universitaria autónoma. Revista Venezolana de Gerencia, 16 (56), 595-607.

Rótulo, D.; M. Baudean, F. Abrucio y N. Cohn. (2014). Gobernanza y políticas de cooperación fronteriza: el caso Brasil-Uruguay. Revista del CLAD, 58, 135-166.

Safari, A. (2012). Customers' international online trust - Insights from focus group interviews. Journal of Theoretical \& Applied Electronic Commerce Research, 7 (2), 59-72.

Sanabria, M.; J. Saavedra y A. Smida. (2013). Los estudios organizacionales ("organization studies"): fundamentos, evolución y estado actual del campo. Bogotá: Universidad del Rosario.

Sánchez, J. (2014). Usos de la accountability en la administración pública. Revista Venezolana de Gerencia, 19 (68), 618-638.

Sánchez, J.; T. Pintado, H. Talledo y S. Carcelén. (2009). La educación de posgrado en España. Un estudio empírico de la estructura de motivaciones latentes. Innovar, 19, 131-140.

Sánchez-Torres, J., and S. Rivera. (2009). A model for measuring research capacity using an intellectual capital-based approach in a Colombian higher education institution. Innovar, 19, 179-197.

Santana, L. (2000). Tendencias curriculares en la enseñanza de la administración pública en los Estados Unidos. Revista del CLAD, 17, 1-17.

Santos, J. e C. Barros. (2011). 0 que determina a tomada de decisão financeira: razão ou emoção. RBGN, 13 (38), 7-20.

Scott, W. (2003). Organizations: Rational, natural, and open systems, $5^{\text {a }}$ ed. Upper Saddle River: Prentice-Hall.

Scott, W. (2004). Reflections on a half-century of organizational sociology. Annual Review of Sociology, 30 (1), 1-21.

Shankar, B. (2009). Theory building for hypothesis specification in organizational studies. London: Sage. 
Shenhav, Y. (2003). The historical and epistemological foundations of organization theory: Fusing sociological theory with engineering discourse. In H. Tsoukas, and C. Knudsen (Eds.), The Oxford handbook of organization theory (pp. 183-209). 0xford-NY: 0xford University Press.

Siegler, J.; C. Biazzin y A. Rodrigues. (2014). Fragmentación del conocimiento científico en administración: un análisis crítico. RAE, 54 (3), 254-267.

Taylor, F. (1961). Principios de administración científica. México: Herrero Hermanos.

Tognato, C. (2007). Construir zonas de intercambio entre la academia y el mercado: una aproximación desde la pragmática cultural. Innovar, 44 (2), 7-18.

Torres, E.; E. Marinao, and C. Chasco. (2012). Development and proposal of a scale for measuring the image of tourist destinations (Imatur). RBGN, 14 (45), 400-418.

Tranfield, D.; D. Denyer, and P. Smart. (2003). Towards a methodology for developing evidence-informed management knowledge by means of systematic review. British Journal of Management, 14 (3), 207-222.

Tsoukas, H., and C. Knudsen. (2005). The Oxford handbook of organization theory. Oxford, UK: 0xford University Press.

Ullah, M.; I. Butt, and M. Haroon. (2008). The journal of Ayub Medical College: A 10 years bibliometric study. Health Information and Libraries Journal, 25 (2), 116-124.

Uribe, J.; C. Ortiz y J. Domínguez. (2011). La rentabilidad de la formación para el empleo en Colombia. Cuadernos de Administración, 24 (43), 111-132.

Velásquez, J.; Y. Olaya y C. Franco. (2009). Evidencias de cambios estructurales en el precio promedio mensual del petróleo del West Texas Intermediate (WTI). Cuadernos de Administración, 22 (38), 247-266.

Velásquez, M.; M. Novoa y M. Mayorga. (2006). La organización escolar: punto de partida de la evaluación de los docentes. Innovar, 16 (28), 93-110.

Vieira, M., e R. da Silva. (2011). Eight propositions towards new possibilities of studying organizing and organizations. BAR, 8 (4), 454-467.

Vizeu, F. (2010). Potencialidades da análise histórica nos estudos organizacionais brasileiros. $R A E, 50$ (1), 37-47.

Wood, T., e A. Paes. (2004). 0 fenômeno dos mpas brasileiros: hibridismo, diversidade e tensões. $R A E, 44(1), 116-129$.

Woodward, J. (1975). Management and technology. In D. Pugh, Organization Theory (pp. 5671). London: Penguin Books.

Wren, D.; M. Buckley, and L. Michaelsen. (1994). The theory/applications balance in management pedagogy: Where do we stand? Journal of Management, 20 (1), 141-157.

Yen-Tsang, C.; J. Csillag, e J. Siegler. (2012). Teoria da ação racional na competência de melhoria contínua: abordagem comportamental. $R A E, 52(5)$, 546-564. 
